This is the final peer-reviewed accepted manuscript of:

Luminescent sensors based on quantum dot-molecule conjugates

Serena Silvi and Alberto Credi

Chem. Soc. Rev., 2015, 44, 4275-4289

The final published version is available online at: https://doi.org/10.1039/C4CS00400K

Rights / License:

The terms and conditions for the reuse of this version of the manuscript are specified in the publishing policy. For all terms of use and more information see the publisher's website.

This item was downloaded from IRIS Università di Bologna (https://cris.unibo.it/)

When citing, please refer to the published version. 


\title{
Luminescent sensors based on quantum dot-molecule conjugates
}

\author{
Serena Silvi ${ }^{\star a}$ and Alberto Credi ${ }^{\star a}$
}

\begin{abstract}
Semiconductor quantum dots (QDs) are inorganic nanoparticles that exhibit unique sizedependent optical and electronic properties; in particular, they are strongly luminescent. Their surface can be chemically modified, by either covalent or non-covalent approaches, in order to interface them with molecular units endowed with specific physical and chemical properties. Photoinduced electron- and energy-transfer processes between quantum dots and attached molecular species offer versatile strategies to modulate the photophysical properties of these nanoassemblies in response to a chemical stimulation. Hence, QD-molecule conjugates are appealing platforms for developing luminescent sensors according to a modular design. In this review we discuss the principles underlying the rational construction of this kind of multicomponent species, and we illustrate selected examples of luminescent QD-based sensors taken from the recent literature.
\end{abstract}

\section{Introduction}

Semiconductor nanocrystals known as quantum dots (QDs) are spherical particles with diameters typically ranging between 1 and $15 \mathrm{~nm}$. Upon photoexcitation of QDs, electron-hole pairs are generated, whose recombination causes the emission of light. Because of the very small size, the optical and electronic properties of QDs are affected by quantum effects, resulting, for example, in size dependent absorption and emission spectra. Since their discovery ${ }^{1}$ in the early 1980 s and the development of solution-based preparation methods affording an accurate size control, ${ }^{2}$ it became clear that these particles could represent a valid alternative to molecular species for luminescence applications ${ }^{3}$ and were rapidly introduced for use in bioimaging and diagnostics. ${ }^{4}$ Indeed, the interest on QDs as alternatives to organic dyes has increased dramatically, as witnessed by the number of papers, reviews ${ }^{5}$ and books ${ }^{6}$ on the topic.

Luminescent chemosensors offer the possibility of a sensitive, rapid and reliable determination of a wide range of analytes using simple and cheap instrumentation. The modular construction of multicomponent molecular sensors based on changes in light emission has been extensively investigated in the past four decades. ${ }^{7}$ Since the surface of QDs can be decorated with molecular species, such nanoparticles can be used as scaffolds for the development of multicomponent nanoscale devices. It is therefore not surprising that in more recent times a lot of attention has been devoted to quantum dots as components of luminescent sensors. ${ }^{8}$ The advantage of using QD-molecule conjugates in place of traditional molecular based sensors resides in the properties of these nanomaterials, some of which are shared with nanoparticles in general, while some others are peculiar of inorganic semiconductor nanocrystals. Nanoparticles are handy platforms to build upon and their versatile modification is combined with a large surface to volume ratio. Furthermore, QDs are characterized by unique optical properties (vide infra), which confer them versatility and sensitivity. Indeed, the preparation of nanocrystal-molecule hybrids and a detailed physico-chemical understanding of nanocrystal-molecule interactions are crucial for extending the basic knowledge on these fascinating systems and foster the design of QDs tailored for specific technological applications.

This Tutorial Review will describe hybrids composed of semiconductor nanocrystals attached to functional molecular units, in which the interplay between the QD and molecular components is exploited to perform luminescence sensing towards a variety of ionic and molecular analytes. It should be noted that chemical or physical interactions of the analyte directly with the QD surface can affect the photophysical properties of the nanocrystal, thus enabling the use of bare QDs for luminescent sensing. ${ }^{9}$ Such an approach, however, appears to be limited to a few small ions or molecules capable of reacting directly with the QD surface, and will not be 
considered here. Similarly, we will not discuss the use of bare QDs as sensors of temperature and pressure.

The first part of the paper will deal with the basic physicochemical properties of quantum dots, present the methodologies for the chemical modification of their surface, and discuss the processes that can be exploited to obtain chemical switching of luminescence in nanocrystal-molecule conjugates. In the second part we will illustrate such concepts by describing examples taken from the recent literature, mostly based on CdSe-ZnS QDs, subdivided according to the target analyte(s). The selected case studies are intended to give an overview of the different systems and mechanisms utilized for the detection of each analyte category. Finally, we will discuss perspectives and limitations of QD-molecule hybrids for luminescent sensing purposes.

\section{Optical and electronic properties of QDs}

Several physical properties of bulk materials change substantially when they are in the form of particles with a size on the order of nanometers. Specifically, for semiconductor nanoparticles the band gap - that is, the energy difference between the valence and conduction band edges - can be changed upon varying the particle size. In a semiconductor, absorption of a photon with energy equal or greater than the band gap results in excitation of an electron from valence to conduction levels, leaving a hole in the valence band. Such an electron-hole pair is bound by the electrostatic attraction between the opposite charges and is called an exciton. The exciton Bohr radius measures the average distance between the photogenerated electron and hole. When the size of the particle approaches that of the exciton (for example, the exciton Bohr radius for $\mathrm{CdSe}$ is about $6 \mathrm{~nm}$ ), the optical and electrical properties of the material become dependent on its physical dimension, owing to quantum confinement effects. ${ }^{1-6}$ In these conditions, the band structure of the semiconductor changes into discrete levels (Fig. 1), and the energy difference between the highest occupied level and the lowest unoccupied level widens as the particle size decreases. In other words, the smaller the particle, the more blue shifted their luminescence (Fig. 2).

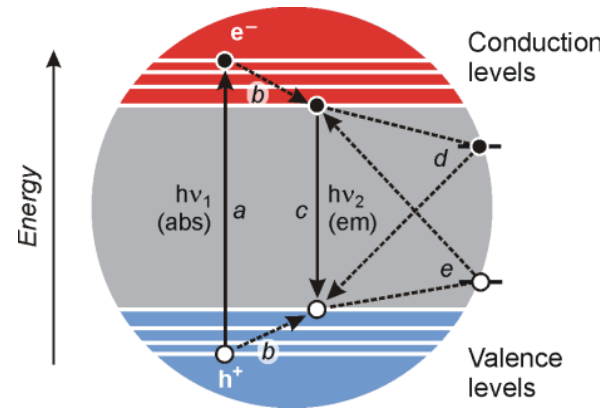

Fig. 1 Schematic representation of important optical and electronic processes in a semiconductor nanocrystal. Solid and dashed lines represent radiative and nonradiative processes, respectively. Legend of processes: $a$, optical excitation; $b$, thermal relaxation of the excited electron and hole; $c$, radiative exciton recombination (luminescence); $d$, nonradiative exciton recombination mediated by an oxidising surface state (electron trap); $e$, nonradiative exciton recombination mediated by a reducing surface state (hole trap).

As discussed above, QDs can absorb photons with energies equal or greater than the band gap energy (process a in Fig. 1). The corresponding absorption spectrum is characterized by a sharp band on the low energy side, corresponding to population of the first exciton state exhibiting a strong oscillator strength (Fig. 3). Its position and intensity depend on the particle size, while its width and shape reflect the size distribution of the QDs. In general quantum dots are characterized by a continous absorption, with large molar coefficients, in the UV-visible region, so that they can be effectively excited in a wide spectral region and with low intensity light. They also exhibit a remarkable cross section for two-photon absorption.

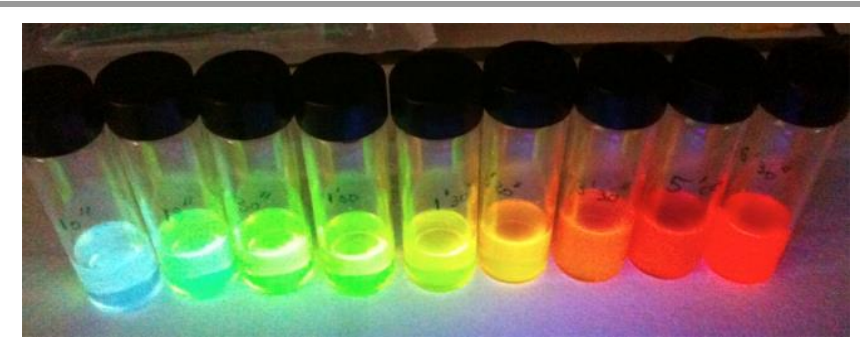

Fig. 2 Photograph of chloroform solutions of CdSe-ZnS QDs of different size (smaller to larger from left to right) under UV excitation.

In direct band gap semiconductors (i.e., in which the lowest energy conduction level and the highest energy valence level have the same momentum), such as $\mathrm{CdS}, \mathrm{CdO}, \mathrm{CdSe}, \mathrm{CdTe}$, $\mathrm{ZnS}, \mathrm{ZnSe}, \mathrm{GaAs}$ and $\mathrm{InP}$, the recombination of the exciton leads to emission of a photon (process $\mathrm{c}$ in Fig. 1). Owing to quantum confinement, even QDs based on indirect bandgap semiconductors (e.g., Si) can exhibit luminescence. The emission of QDs is typically very intense and, in contrast with most organic fluorophores, it occurs with a remarkably narrow and symmetric spectral profile (Fig. 3). The full width at half maximum (fwhm) is typically on the order of $30 \mathrm{~nm}$, and energy distance between the absorption and luminescence band maxima (Stokes shift) is remarkably small. The emission energy can be adjusted to fall in a spectral region ranging from the UV to the IR, by selecting the chemical composition (i.e., the band gap of the bulk material) and the size of the particles (to take advantage of quantum confinement).

It should be noted that, in order to have an efficient emission, the surface of the nanocrystals must be appropriately passivated; otherwise, the charge carriers would be most likely trapped in surface states and undergo nonradiative recombination (processes $d$ and e in Fig. 1). As a matter of fact, the presence of surface states, originating from the dangling bonds of the surface atoms, crystal defects and/or impurities, have a profound influence on the spectroscopic and redox properties of QDs. ${ }^{6}$ The luminescence quantum yield of CdSe nanocrystals is typically in the $10-25 \%$ range but it strongly depends on the nature of surface ligands. ${ }^{10}$ For example CdSe prepared using long chain primary amines are found to have emission quantum yields of up to $60 \%$. Indeed, the 
luminescence quantum yield can be improved by coating the nanocrystal with a layer of another semiconductor material having a larger bandgap. Nanocrystals of this type are known as core-shell quantum dots. ${ }^{11}$

CdSe is a frequently used semiconductor to make QDs for luminescence applications because size modulation of its band gap enables an accurate tuning of the emission maximum across the visible region (Fig. 2). For example, on changing the diameter of CdSe QDs from 2.6 to $3.1 \mathrm{~nm}$ the wavelength of the luminescence maximum moves from 540 to $570 \mathrm{~nm} .{ }^{12} \mathrm{CdSe}$ nanocrystals can be efficiently passivated by depositing a shell of $\mathrm{ZnS}$; indeed, CdSe-ZnS core-shell particles are the most popular $\mathrm{QDs}^{5 \mathrm{c}}$ and are commercially available in various sizes. Not surprisingly, the vast majority of the examples found in the literature, as well as those described in this article, are based on CdSe-ZnS QDs.

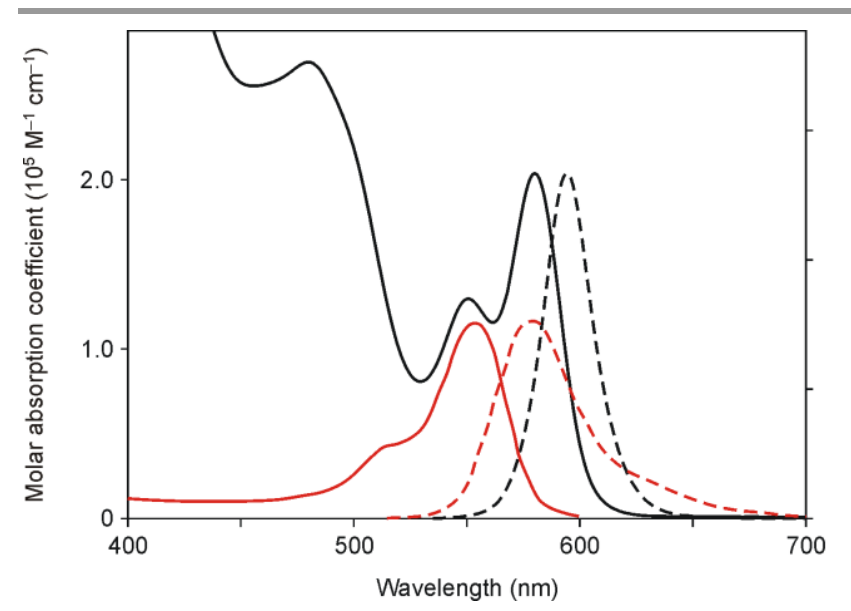

Fig. 3 Absorption (full line) and photoluminescence (dashed line; $\lambda_{\text {exc }}=500 \mathrm{~nm}$ ) spectra of CdSe QDs $\left(d=3.8 \mathrm{~nm}\right.$; black curves, $\left.\mathrm{CHCl}_{3}\right)$ and of the well known dye Rhodamine $\mathrm{B}$ (red curves, $\mathrm{MeOH}$ ) at room temperature.

The luminescence lifetimes of QDs at room temperature are usually comprised between 10 and 40 ns. These values are long enough to enable time-gated detection in bioimaging experiments, which reduces the short-lived fluorescence signal arising from many biomolecules. However, as they still fall in the ns timescale, high count rates can be obtained in time-gated measurements, thus avoiding the detection limitations encountered with long-lived luminophores such as lanthanides complexes.

Another important property of QDs is their resistance to photobleaching, which is much higher than that of molecular fluorophores, in particular for core-shell systems; therefore, quantum dots are particularly fit for single-particle detection or in applications that require irradiation with high intensities and/or long periods.

\section{Preparation and surface functionalization of the nanocrystals}

Although direct synthesis of semiconductor nanocrystals in water solution is possible ${ }^{13}$ (e.g. using thioglycolic acid as a capping agent), ${ }^{14}$ in most instances QDs are prepared in apolar organic solvents by reacting inorganic precursors in the presence of molecular ligands forming a coating that stabilizes the nanoparticle surface. As mentioned above, a shell of another semiconductor with a larger band gap can be deposited onto the surface of the core nanocrystal in order to improve its optical properties. Such synthetic methods enable an excellent control over size, shape and composition, and afford highly hydrophobic QDs whose surface is typically covered with alkylphosphine, alkylamine and/or alkanethiol ligands. ${ }^{10}$ These hydrophobic particles can be successively transferred into aqueous solutions by, e.g., incorporation in a polymer shell or in micelles. ${ }^{15}$

It should be noted, however, that the preparation protocols of QDs, unlike molecular syntheses, are extremely sensitive to conditions, and are often characterized by reproducibility issues or strong dependence on the quality of the precursors and solvents. ${ }^{5 e, 6,8 e, 16}$ This means that the same synthetic procedure can yield QDs with different characteristics (e.g., luminescence quantum yield) even if repeated by the same person in the same laboratory. Indeed, additional standardization efforts, in comparison with molecular chemistry, have to be made. When performing experiments, researchers should also be aware of the fact that in materials chemistry reproducing results may not be straightforward.

A key aspect of most ligands is the dynamic nature of their binding to the nanoparticle surface, thereby allowing the exchange of the native ligands with other surfactants bearing different functionalities. For example, biological uses require that the QD surface be engineered in order that the particle is soluble in water. Fig. 4 represents some strategies developed to make water soluble QDs which can also be applied to decorate QDs with functional molecular units via the covalent or noncovalent attachment of such units to the water-solubilizing ligands. ${ }^{8,17}$ Chemically functionalized QDs soluble in organic solvents can also be prepared, usually by replacing the native ligands with suitably functionalized molecular ligands.

Drawbacks of surface modification methodologies include instability of the molecular coating, degradation of the optical properties, QD aggregation and poor versatility. A common problem for these strategies is the increase of the final size of the QDs, posing limitations to their use in biological applications. In order to obtain compact, biocompatible nanocrystals, the coverage of QDs by simple carbohydrates separated from the inorganic core through a polyethylenglycol (PEG) spacer was proposed (Fig. 4d). ${ }^{18}$ Polymeric ligands with multiple thiol groups, poly(ethylene glycol) chains, and reactive functional groups appended to a common macromolecular backbone were also reported. ${ }^{19}$ The thiol groups displace the native hydrophobic ligands of the QD and afford a stable anchoring of the polymers onto the nanocrystal surface; the poly(ethylene glycol) chains impose hydrophilic character and the reactive groups enable the conjugation with other molecular units. CdSe-ZnS QDs coated with such polymeric ligands exhibit valuable properties in terms of stability over a broad $\mathrm{pH}$ range, water solubility and luminescence quantum yield, and show a hydrodynamic diameter on the order of $15 \mathrm{~nm} .{ }^{19}$ 
QDs have generally a little intrinsic value as luminescent sensors because their photophysical properties are not altered by the presence of analytes in the surrounding medium (with the exception of metal ions that can exchange with those on the nanocrystal surface, as mentioned in the Introduction). This is particularly true for strongly emissive nanocrystals, i.e., those that can afford high sensitivity in luminescent measurements. In fact, QDs that exhibit large emission quantum yield are characterized by a very efficient passivation of their surface (by an inorganic coating and/or the monolayer of capping ligands), thus rendering them insensitive to the surrounding environment. ${ }^{4-6,8,11}$ Hence, a strategy to implement a sensing response with QDs is to combine them with chemosensitive molecular components.
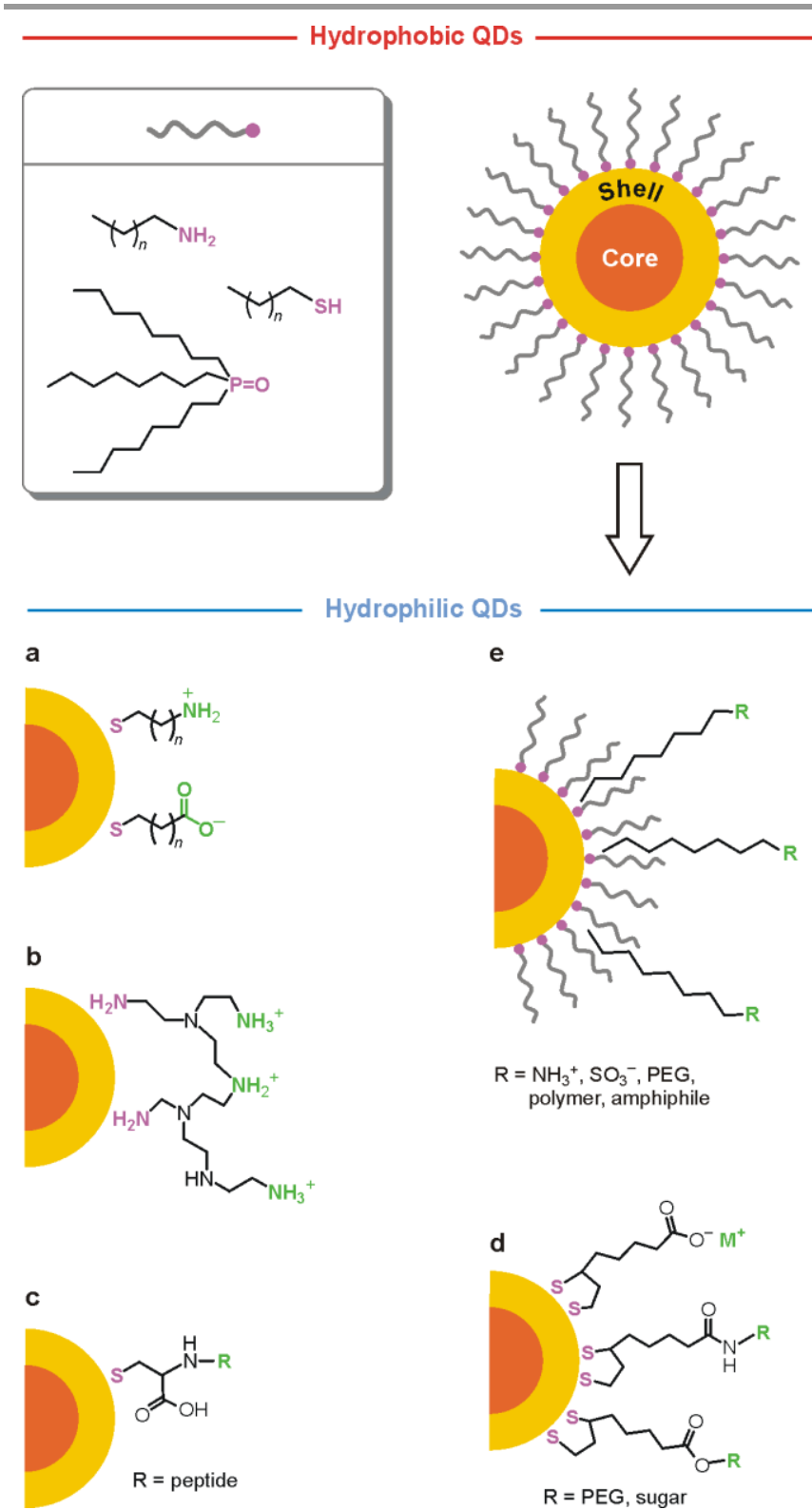

$\mathrm{R}=\mathrm{NH}_{3}{ }^{+}, \mathrm{SO}_{3}{ }^{-}, \mathrm{PEG}$, polymer, amphiphile

Fig. 4 Strategies for the modification of the surface of hydrophobic QDs for functionalization and/or water solubilization and functionalization. Ligand exchange with (a) mercaptoammonium or mercaptocarboxylic derivatives, (b) polyamines, (c) cysteine-modified peptides, (d) dithiolane (lipoic acid) derivatives. (e) Encapsulation of the hydrophobic QD by self-assembly with amphiphilic cations, anions or polymers (PEG = polyethylene glycol).

\section{Light-induced interplay between quantum dots and molecular components}

The photoinduced exchange of electronic energy and electrons between connected molecular components is commonly used to obtain light-triggered functionalities in multicomponent (supramolecular) systems. $^{20}$ Examples of artificial lightharvesting antennas, wires, connectors, switches, sensors, logic gates, memories, and molecular machines obtained following this approach are available. ${ }^{21}$

Generally speaking, the energy levels of the components must be correctly positioned relatively to one another in order for these processes to be thermodynamically allowed. For example, if the QD is in contact with a molecular electron acceptor exhibiting a LUMO level at a lower energy than that of the conduction band edge, a photoinduced electron transfer from the QD to the electron acceptor can take place (Fig. 5a), with consequent quenching of the QD luminescence. Conversely, if the nanocrystal is linked to an electron donor possessing a HOMO level at a higher energy with respect to the valence band edge, an electron transfer from the molecular donor to the photoexcited QD - or, alternatively, hole transfer from the QD valence band to the molecular HOMO - can occur (Fig. 5b).
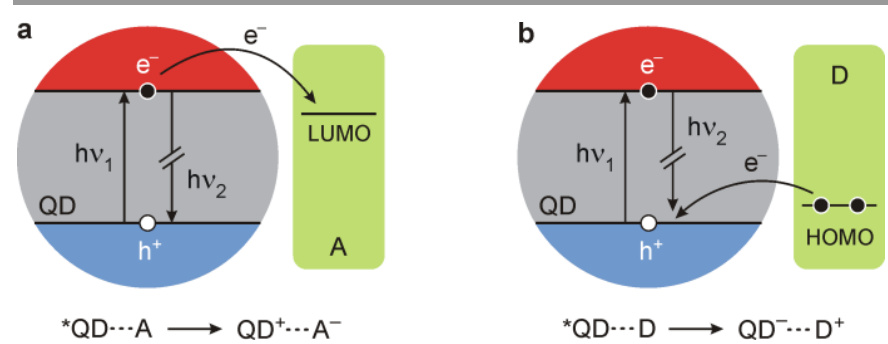

Fig. 5 Schematization of (a) electron transfer from a photoexcited QD to an electron acceptor $A$, and (b) electron transfer from an electron donor $D$ to $a$ photoexcited QD. Adapted by permission from ref. 5h. Copyright 2014 Elsevier.

The free energy for the photoinduced electron transfer processes shown in Fig. 5 can be estimated with eq. 1:22

$\Delta G_{e T}^{0}=e E_{\mathrm{D}^{+} \mathrm{D}}-e E_{\mathrm{AA}^{-}}-e E_{0-0}-\frac{e^{2}}{4 \pi \varepsilon_{0} \varepsilon_{\mathrm{r}} d}$

in which $e$ is the electron charge, $E_{\mathrm{D}+/ \mathrm{D}}$ is the potential for oxidation of the species acting as the electron donor (QD in Fig. $5 \mathrm{a}$, molecule D in Fig. 5b), $E_{\mathrm{A} / \mathrm{A}^{-}}$is the potential for reduction of the species acting as the electron acceptor (molecule $\mathrm{A}$ in Fig. 5a, QD in Fig. 5b), $E_{0-0}$ is the energy change of the transition caused by the photoexcitation of the QD, $\varepsilon_{0}$ is the vacuum permittivity, $\varepsilon_{r}$ is the dielectric constant of the medium, and $d$ is the luminophore-quencher distance. The application of eq. 1 to nanocrystal-molecule conjugates, however, is not straightforward because the redox properties of QDs are often 
dominated by surface traps and thus are not related to valence and conduction band levels. ${ }^{12}$

Another common quenching pathway involves the transfer of electronic energy between the QD and a nearby molecular component. In the case of nanocrystal-molecule conjugates, energy transfer usually takes place by a dipole-dipole (Förster) mechanism, which is frequently referred to as fluorescence resonance energy transfer (FRET, Fig. 6). In most instances, QD-molecule hybrids are designed such that the electronic energy is transferred from the photoexcited QD to the molecular chromophore (Fig. 6a). ${ }^{8}$ Systems exhibiting energy transfer in the reverse direction - that is, from a photoexcited molecule to the QD (Fig. 6b) - are less common. ${ }^{3 b}$ Indeed, the observation of the photosensitized luminescence of the nanocrystal (hv 4 in Fig. 6b) is complicated by the fact that the very intense and broad absorption spectrum of the QD usually prevents a selective, substantial excitation of the attached molecule.

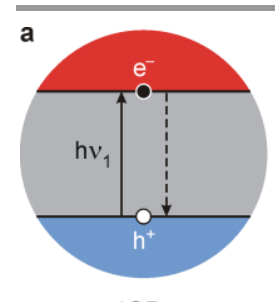

${ }^{\star} \mathrm{QD}$

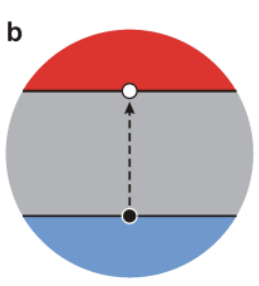

QD

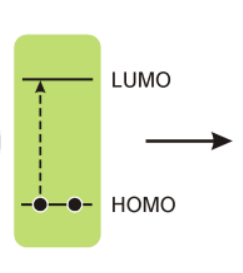

A

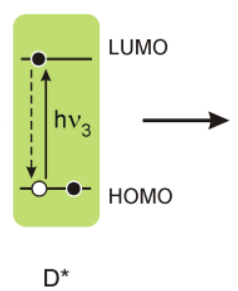

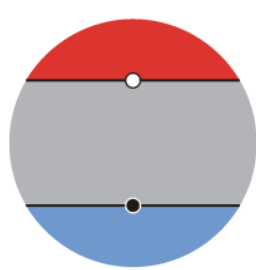

QD

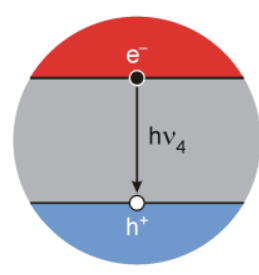

QD*

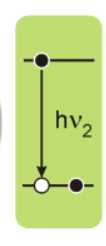

${ }^{*} A$

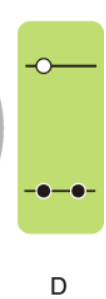

Fig. 6 Schematization of dipole-dipole energy transfer (FRET) (a) from a photoexcited QD to a molecular energy acceptor (A), and (b) from a photoexcited molecular energy donor (D) to a QD. The energy-transfer process may give rise to photosensitized emission of the energy acceptor component $\left(h v_{2}\right.$ for a, hv $v_{4}$ for b). Reproduced by permission from ref. 5h. Copyright 2014 Elsevier.

A detailed description of the mechanisms of energy- and electron-transfer processes is beyond the scope of this article and can be found elsewhere, ${ }^{21,23}$ however, one can generically say that the rate of these processes depends on various parameters of the partners such as their spectral features, distance, electronic coupling, and spin properties. If one or more of these parameters is affected by stimulation, a switching behaviour can be obtained.

Luminescence switches are species in which a change in one or more input signals results in a change in photoluminescence output. ${ }^{7,21}$ A modular design of a luminescent switch requires the presence of three components: the luminophore (L) - a unit capable of emitting light upon photoexcitation, the quencher $(\mathrm{Q})$ - a unit capable of quenching the luminophore emission by some mechanism, and the control component - a unit capable of affecting the behaviour of the quencher in response to external stimulation. In the case of luminescent chemosensors (Fig. 7) the control component is a receptor $(\mathrm{R})$ that can recognize the target analyte $(\mathrm{A})$; the binding of the analyte to the receptor influences the ability of the quencher component to alter the emission of the luminophore. ${ }^{7}$ It should also be noted that in many instances the quenching and controlling functions described above can be performed by the same chemical component. Hence, a luminescent chemosensor can also be viewed as a switch controlled by a chemical input, the latter being coded for by a change in the analyte concentration. Luminescent switches that respond to either electrochemical or photochemical inputs have also been designed and investigated. An objective of this research is the construction of 'intelligent' systems that implement binary logic functions. ${ }^{21}$

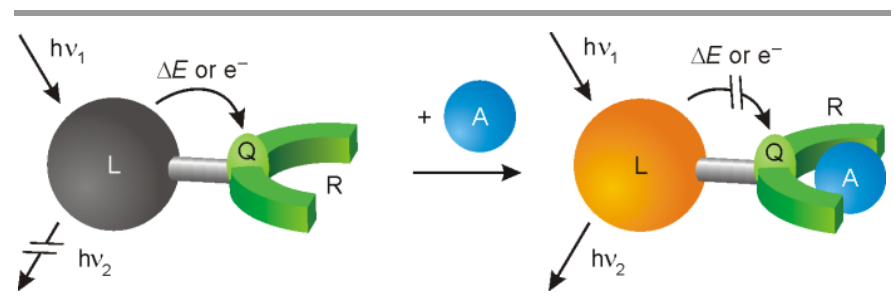

Fig. 7 Scheme of the analyte-modulated "turn on" photoluminescence response in a luminophore-quencher-receptor multicomponent chemosensor.

These design strategies have been successfully applied to develop a large variety of photochemical molecular devices, taking advantage of the huge amount of information available on the spectroscopic and redox properties of molecular species. In principle, the very same operating mechanisms can be extended from organic dyes to quantum dots, in order to enable the modular construction of systems containing both molecular and nanocrystal components. ${ }^{24}$ QDs possess indeed suitable optical and electronic features to become involved in energyand electron-transfer phenomena. However, the level of understanding reached on the interplay between the structural and electronic properties of molecular species has not yet been achieved in the case of quantum dots. For example, a rationalization of the effect of the diameter of their luminescent core, the thickness of their protective shell and the nature of their passivating ligands on their redox potentials and electron transfer kinetics is often not straightforward. Indeed, systematic investigations on the structural factors regulating the redox properties of quantum dots are still relatively rare $^{5 \mathrm{e}}$ and indicate that surface defects play a major role in determining the redox properties of QDs (Fig. 1), thereby complicating the rational design of multicomponent systems whose function is based on electron-transfer processes.

Nevertheless, conventional formalisms for the interpretation of energy- and electron-transfer processes between molecules have been successfully applied to quantum dots, and several examples of luminescent sensors based on the interplay between quantum dots and surface-bound functional molecular species have been reported. Representative examples, 
subdivided according to the kind of target analytes, are described in the next sections.

\section{pH Sensors}

$\mathrm{pH}$ is a most important target parameter for applications ranging from environmental control to medical diagnostics.

The QDs surface ligands are ususally Lewis bases, and as such they are sensitive to acid environments. ${ }^{25}$ Nevertheless in a more popular methodology for $\mathrm{pH}$ sensing a fluorophore with a known pH-response is conjugated to the QD surface. ${ }^{26}$

An early example of a $\mathrm{pH}$ sensitive QD-molecule hybrid is shown in Fig. 8. ${ }^{27}$ The sensing is based on energy and electrontransfer processes between CdSe-ZnS quantum dots and $[1,3]$ oxazine ligands adsorbed on the surface of the nanocrystals. Upon addition of either base or acid to the ligand, the [1,3]oxazine ring opens to generate a 4nitrophenylazophenolate or 4-nitrophenylazophenol chromophores, respectively, which exhibit peculiar electrochemical and spectroscopic properties. o
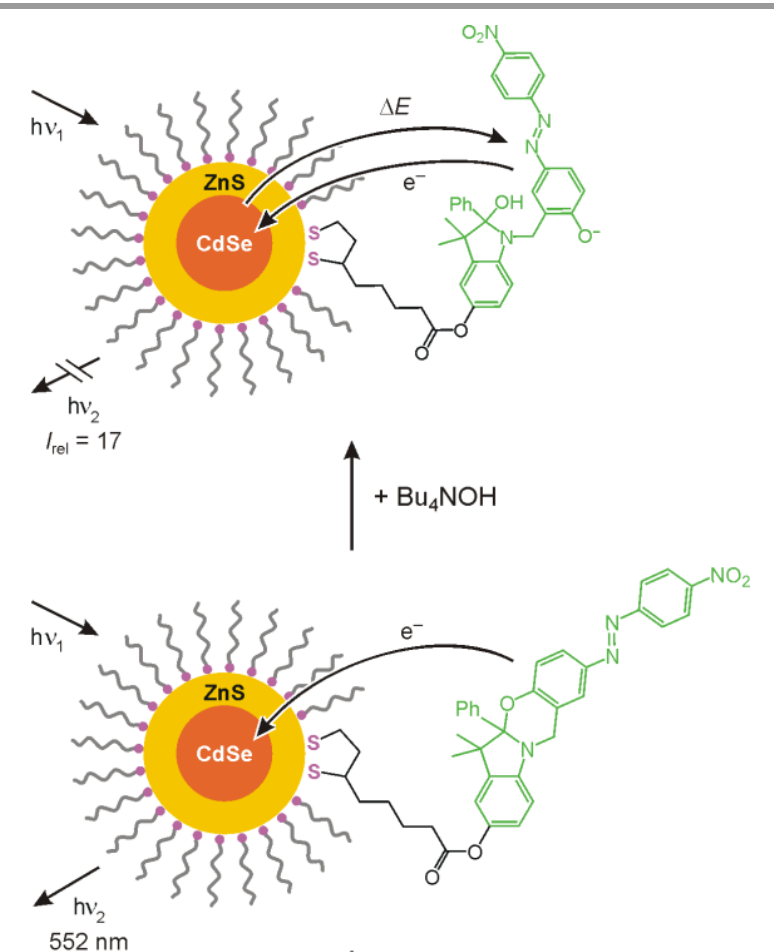
$552 \mathrm{~nm}$
$I_{\text {rel }}=100$

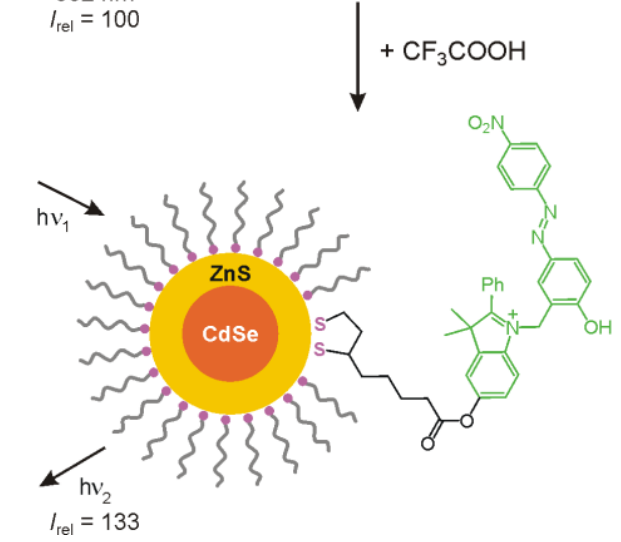

Fig. 8 A luminescent $\mathrm{pH}$ sensor based on CdSe-ZnS QDs modified with a $[1,3]$ oxazine ligand with $\mathrm{pH}$-dependent electrochemical and spectroscopic properties. ${ }^{27}$

The ligand in its closed form absorbs in the UV region and is oxidized at $+0.88 \mathrm{~V}$ vs $\mathrm{Ag} / \mathrm{AgCl}$. Upon formation of the QDligand conjugate, the emission of the QDs $\left(\lambda_{\mathrm{em}}=552 \mathrm{~nm}\right)$ is quenched by electron transfer from the ligand to the QD. On addition of $\mathrm{Bu}_{4} \mathrm{NOH}$, the 4-nitrophenylazophenolate chromophore is more easily oxidized and absorbs in the range of wavelengths where the quantum dots emit. Therefore, the base-triggered transformation of the ligands increases the efficiency of the electron-transfer from the organic to the inorganic component and enables energy transfer from the quantum dot to the ligand. The overall result is a decrease in the luminescence quantum yield of $83 \%$ (Fig. 9, left).

On the other hand, the 4-nitrophenylazophenol chromophore formed on addition of $\mathrm{CF}_{3} \mathrm{COOH}$ does not absorb in the visible region and is oxidized at more positive potential. Hence, both energy and electron-transfer processes are inefficient and the luminescence quantum yield increases by 
33\% (Fig. 9, left). These changes can be exploited to probe the $\mathrm{pH}$ of aqueous solutions in biphasic systems wherein a $\mathrm{CHCl}_{3}$ solution of QD-ligand conjugates and $\mathrm{Bu}_{4} \mathrm{NCl}$ is in contact with an aqueous solution. $\mathrm{A} \mathrm{pH}$ increase from 3.2 to 10.7 in the aqueous phase translates into a luminescence decrease of ca. $29 \%$ in the organic phase (Fig. 9, right).
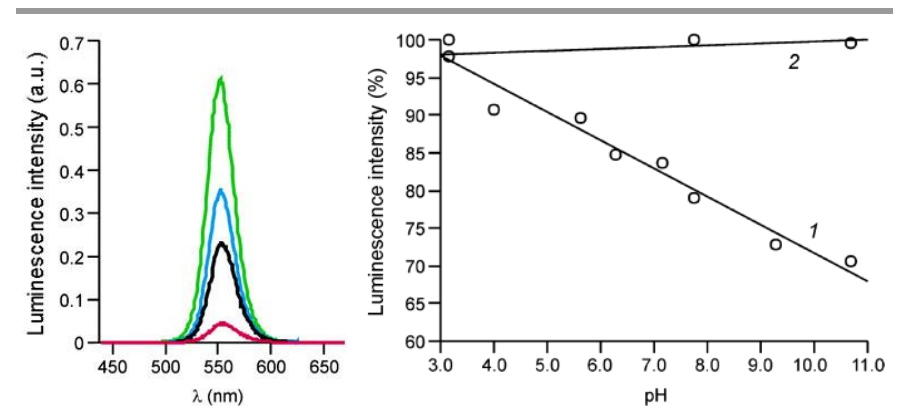

Fig 9. Left: emission spectra of CdSe-ZnS core-shell quantum dots $\left(0.9 \mu \mathrm{M}, \mathrm{CHCl}_{3}\right.$, $20{ }^{\circ} \mathrm{C}, \lambda_{\text {exc }}=426 \mathrm{~nm}$ ) before (green) and after (black) the attachment of the ligand to their surface and the subsequent addition of either $\mathrm{Bu}_{4} \mathrm{NOH}$ (red, 2.5 $\mathrm{mM}$ ) or $\mathrm{CF}_{3} \mathrm{COOH}$ (blue, $2.5 \mathrm{mM}$ ). Right: emission intensity of CdSe-ZnS core-shell quantum dots $\left(0.4 \mu \mathrm{M}, \mathrm{CHCl}_{3}, 0.1 \mathrm{M} \mathrm{Bu} \mathrm{NCl}, 20^{\circ} \mathrm{C}, \lambda_{\text {exc }}=430 \mathrm{~nm}, \lambda_{\text {em }}=552 \mathrm{~nm}\right)$ with (1) and without (2) the ligand on their surface after the addition of sodium phosphate buffer with $\mathrm{pH}$ ranging from 3.2 to 10.7. Adapted with permission from ref. 27. Copyright 2006 American Chemical Society.

The first ratiometric quantum dot $\mathrm{pH}$ sensor is based on a QD-squaraine conjugate (Fig. 10a). ${ }^{28} \mathrm{CdSe}-\mathrm{ZnS}$ nanocrystals were encapsulated within an amphiphilic polymer, upon which the squaraine dye was conjugated. The photoexcited nanocrystals may either luminesce $\left(\lambda_{\mathrm{em}}=613 \mathrm{~nm}\right)$ or transfer their excitation energy to the squaraine dye, whose absorption profile depends on the $\mathrm{pH}$ (Fig. 10b, inset). At acidic $\mathrm{pH}$ the absorption band of the dye matches the emission band of the QD; FRET from the QD to the organic ligand is efficient and the emission spectrum of the nanohybrid is dominated by the fluorescence band of the dye at $650 \mathrm{~nm}$. Under basic conditions the absorption band of the squaraine is suppressed, energy transfer from the QD is inefficient, and the emission spectrum of the conjugate shows only the QD band at $613 \mathrm{~nm}$ (Fig. 10b). Therefore, the FRET efficiency is modulated by the $\mathrm{pH}$ of the environment, and the ratio of quantum dot to dye emission becames a function of $\mathrm{pH}$.

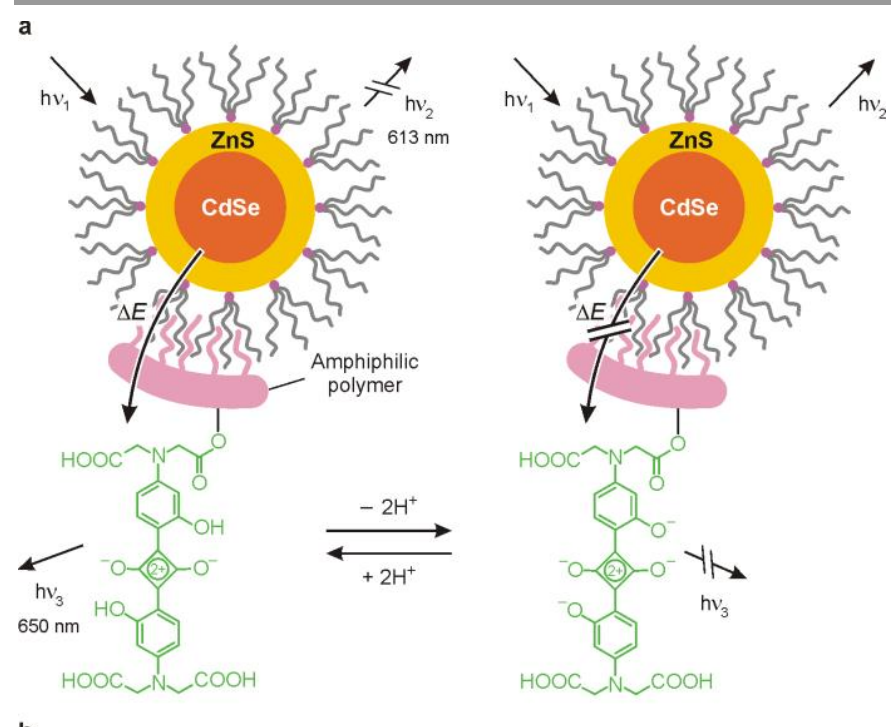

b

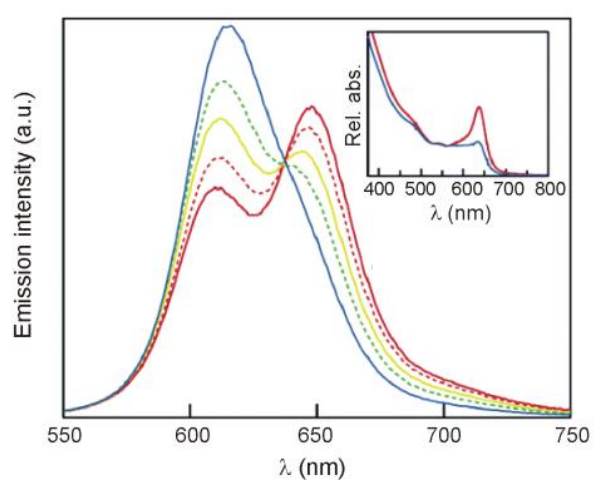

Fig. 10 (a) Scheme of a luminescent pH sensor based on CdSe-ZnS QDs derivatized with a squaraine ligand exhibiting acid-base dependent absorption properties. ${ }^{28}$ (b) Emission spectra of the QD-squaraine conjugate as a function of $\mathrm{pH}\left(\mathrm{H}_{2} \mathrm{O}, \lambda_{\text {exc }}=380 \mathrm{~nm}\right.$ ): 6.0 (red solid line); 7.0 (orange dotted line); 8.0 (yellow solid line); 9.0 (green dotted line); 10 (blue solid line). The inset shows the absorption spectra of the QD-squaraine hybrid at acidic (red line) and basic (blue line) $\mathrm{pH}$ values. Adapted with permission from ref. 28. Copyright 2006 American Chemical Society.

The measurement of $\mathrm{pH}$ has a crucial biological impact, as its regulation is relevant for most cellular processes and plays a critical role in the operation of the cell. In this framework, near infrared (NIR) fluorescent probes are particularly valuable, as this light has the maximum depth of penetration in tissues. CdTeSe-ZnS QDs were conjugated with a carbocyanine dye (Fig. 11), ${ }^{29}$ which exhibits an absorption maximum in the NIR $(750 \mathrm{~nm})$ at low $\mathrm{pH}$ that undergoes a hypsochromic shift to the visible region $(520 \mathrm{~nm})$ on increasing $\mathrm{pH}$. Two different QDs, emitting either at $520 \mathrm{~nm}$ or $750 \mathrm{~nm}$ (QD560 and QD 750 , respectively), were labelled with the dye with the purpose of matching the absorption shift of the dye at different $\mathrm{pHs}$. The luminescence band of QD 750 has an excellent overlap with the NIR absorption of the dye at acid $\mathrm{pH}$, whereas that of QD560 overlaps the dye absorption band at neutral or basic $\mathrm{pH}$.

In order to determine the FRET efficiency, the authors investigated the $\mathrm{pH}$ dependence of the luminescence lifetime. As expected from the spectral features of the components, the two nanohybrids exhibit opposite trends as a funtion of $\mathrm{pH}$. 
Indeed, the lifetime of the $\mathrm{QD}_{750}$-dye conjugate decreases from $29 \mathrm{~ns}$ at $\mathrm{pH}>7$ to $12 \mathrm{~ns}$ at $\mathrm{pH}<5$, whereas the lifetime of the QD 560-dye hybrid increases from $7 \mathrm{~ns}$ at $\mathrm{pH}>7$ to $31 \mathrm{~ns}$ at $\mathrm{pH}$ $<5$. The corresponding plots of the lifetime versus $\mathrm{pH}$ (Fig. 12) show a sigmoidal transition with a $\mathrm{pK}_{\mathrm{a}}$ of 5.2 and 5.5, respectively. The combination of the complementary response of the two nanohybrids can be used for ratiometric $\mathrm{pH}$ sensing.
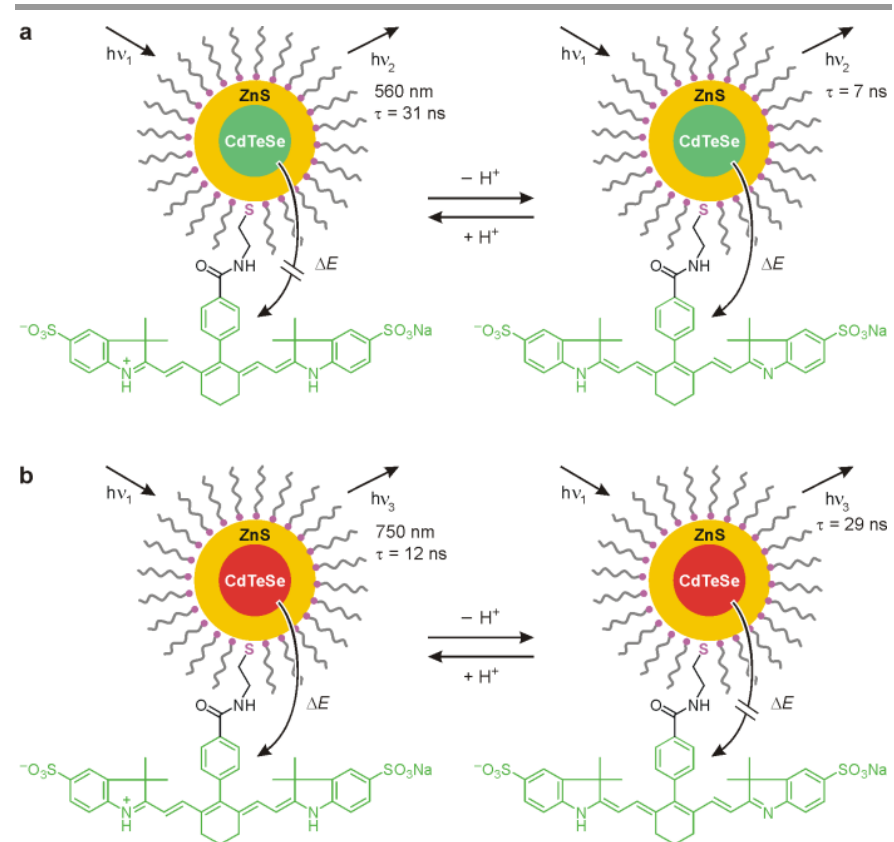

Fig. 11 Schematic representation of the $\mathrm{pH}$ modulation of the luminescence lifetime of two different $Q D$-carbocyanine dye conjugates $\left(a, Q_{560} ; b, Q D_{750}\right) .{ }^{29}$

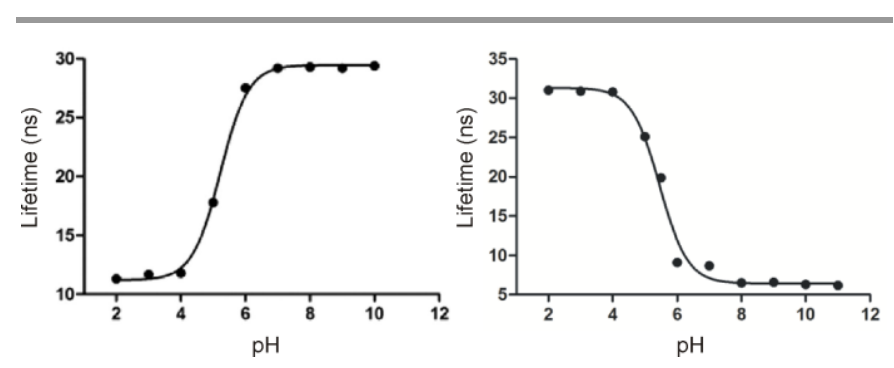

Fig. 12 Sigmoidal fit of the fluorescence lifetime vs $\mathrm{pH}$ curve of the $Q D_{750}$-dye conjugates (left) and of the $\mathrm{QD}_{560}$-dye conjugates (right). Adapted with permission from ref. 29. Copyright 2012 American Chemical Society.

The NIR fluorescence lifetime technique for $\mathrm{pH}$ sensing takes particular advantage from the use of QDs with respect to organic fluorephores. Major limitations of NIR organic dyes are their photostability and the small changes in lifetimes between acid and basic environments. This general strategy combines the excellent photophysical properties of QDs with the high $\mathrm{pH}$ sensitivity of NIR organic dyes.

Intracellular $\mathrm{pH}$ sensing was demonstrated ${ }^{30}$ using CdSe$\mathrm{ZnS}$ QD-dopamine bioconjugates, in which luminescence quenching is caused by an electron-transfer process from the photoexcited QD to an oxidized dopamine-quinone moiety. At low $\mathrm{pH}$, the dopamine is in the reduced hydroquinone form, which produces only marginal quenching of the QD emission $\left(\lambda_{\mathrm{em}}=520,550\right.$ or $\left.580 \mathrm{~nm}\right)$. As $\mathrm{pH}$ increases, ambient $\mathrm{O}_{2}$ oxidizes dopamine to quinone, which is an electron acceptor and significantly quenches the QD emission (Fig. 13). The linear dependence of quenching efficiency versus $\mathrm{pH}$ is similar to that exhibited by the reduction potential of dopamine. Quenching is also accompanied by a shortening of the QD lifetime, and is dependent on the nanocrystal size (the smaller the particle, the larger the quenching).

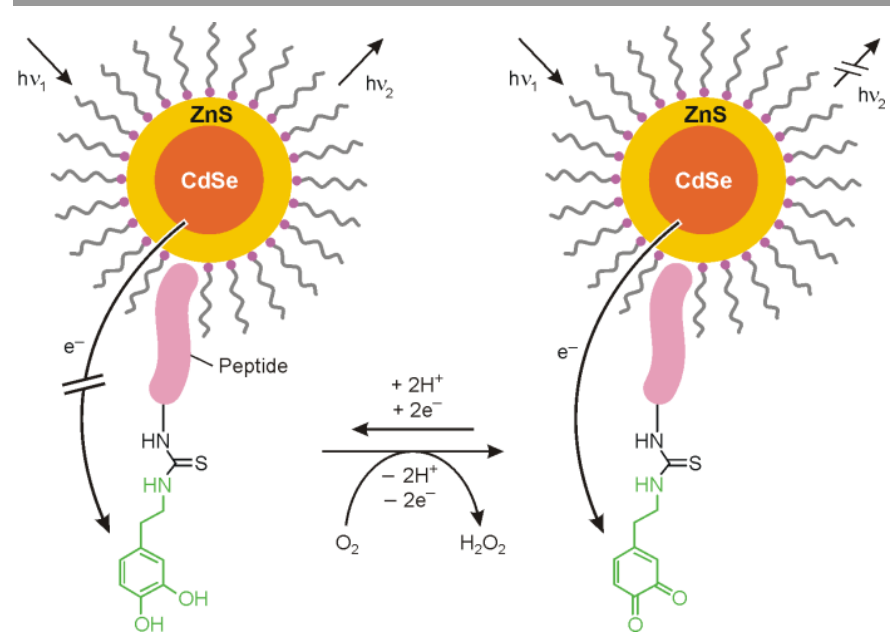

Fig. 13 Peptide-dopamine species are self-assembled on the surface of CdSe-ZnS QDs: the $\mathrm{pH}$ dependent hydroquinone to quinone oxidation triggered by ambient $\mathrm{O}_{2}$ modulates the efficiency of FRET and is exploited to sense $\mathrm{pH}$ in aqueous solution and biological environment. ${ }^{30}$

The QD-dopamine conjugate can also monitor intracellular $\mathrm{pH}$ changes. Cells were injected with the QD-dopamine species at acidic $\mathrm{pH}$, the growth medium was switched to basic $\mathrm{pH}$ in presence or absence of nystatin (a drug that induces pore formation in membranes). A quenching of cytoplasmic QD luminescence in the presence of nystatin was observed as equilibrium was reached between the cytosolic $\mathrm{pH}$ and the surrounding medium.

Another approach for $\mathrm{pH}$ intracellular ratiometric sensing was demonstrated with QD-fluorescent protein conjugates engineered to exhibit FRET processes (Fig. 14). ${ }^{31}$ As the absorption and emission spectra of the fluorescent proteins depend on the $\mathrm{pH}$, the spectral overlap of the FRET pairs, and thus the efficiency of energy transfer, directly correlates to the $\mathrm{pH}$ of the environment and exhibits maximum sensitivity near the $\mathrm{pK}_{\mathrm{a}}$ of the acceptors. The absorbtion band of the protein shifts from $420 \mathrm{~nm}$ at acidic $\mathrm{pH}$ to $550 \mathrm{~nm}$ at basic $\mathrm{pH}$. The fluorescence band of the protein has a maximum at around 570 $\mathrm{nm}$, whereas the QDs emission is centred at $525 \mathrm{~nm}$.

At alkaline $\mathrm{pH}$ values, the sensitized fluorescence emission of the protein upon excitation of the QD indicates that an efficient energy transfer takes place. Upon decreasing $\mathrm{pH}$, the changes in the protein absorbance disable the energy transfer and consequently the QD emission intensity increases while the protein fluorescence decreases. Live cell fluorescence microscopy was performed to image $\mathrm{pH}$ changes temporally and spatially: as the probe progressed through the endocytic pathway the FRET efficiency decreased as a consequence of the drop in $\mathrm{pH}$. 


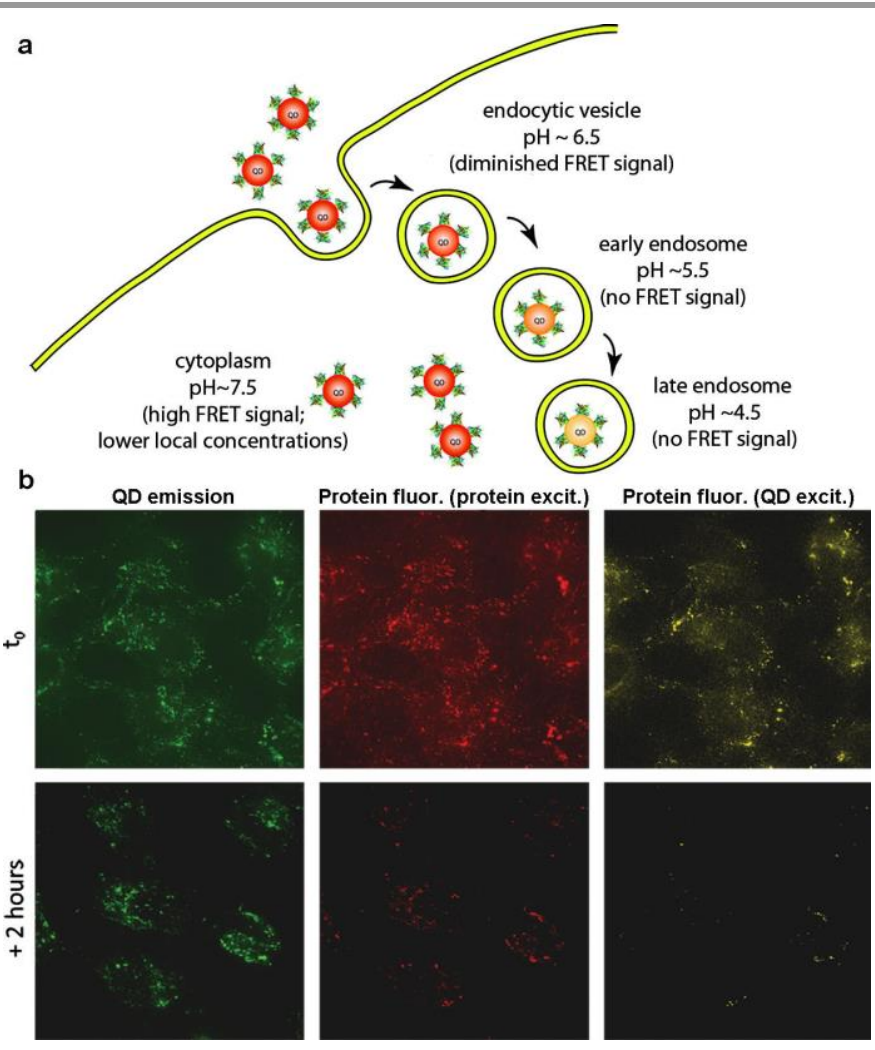

Fig. 14 Fluorescence microscopy cellular imaging of QD-fluorescent protein conjugates exhibiting ratiometric $\mathrm{pH}$ sensing. (a) Schematic representation of the progression of the probe through the endocytic pathway. The FRET efficiency is high in the neutral $\mathrm{pH}$ of the extracellular environment and early endosome. The efficiency decreases as the endosome matures and the endosomal $\mathrm{pH}$ drops, resulting in a diminished fluorescence from the protein and a recovery of the QD emission. Any conjugates that escapes the endosome regains its high FRET efficiency in the pH-neutral cytoplasm. (b) Fluorescence microscopy images immediately after delivery of the QD-protein species and two hours postdelivery. The QD emission (left) demonstrates the consolidation of the probe in the endosomes over time; the images of the protein fluorescence upon direct (center) or sensitized (QD) excitation (right) indicate a decrease in the protein fluorescence and FRET efficiency with maturation of the endosome. Reproduced with permission from ref. 31. Copyright 2012 American Chemical Society.

\section{Sensors for metal cations}

In a pioneering study, metal-ion modulated FRET between QDs of different colours was exploited to detect $\mathrm{K}^{+}$ions at the $\mu \mathrm{M}$ level in aqueous solution. ${ }^{32}$ Two types of CdSe-ZnS QDs with different core sizes $\left(3.2 \mathrm{~nm}, \lambda_{\mathrm{em}}=545 \mathrm{~nm}\right.$, and $5.6 \mathrm{~nm}, \lambda_{\mathrm{em}}=$ $635 \mathrm{~nm}$ ) were functionalized with 15 -crown-5 receptors using a lipoic acid linker. In the presence of $\mathrm{K}^{+}$sandwich complexes involving two receptors and one metal ion are formed; in these complexes, differently sized quantum dots become close enough together to cause energy transfer from the smaller to the larger ones. A ratiometric response for potassium ions was therefore realized, with the emission signals at 545 and $635 \mathrm{~nm}$ decreasing and increasing, respectively, upon addition of $\mathrm{K}^{+}$.

Optical chemosensors obtained by attaching chromogenic Schiff-base receptors to the surface of CdSe-ZnS QDs were investigated. ${ }^{33}$ Interestingly, the selectivity of the receptors was found to be altered upon their incorporation onto the QD surface, most likely because of the spatial organization imparted to the receptors by the three-dimensional surface of the nanoparticle. The simultaneous determination of the concentration of $\mathrm{Cu}^{2+}$ and $\mathrm{Fe}^{3+}$ in buffered solution at physiological $\mathrm{pH},{ }^{33 \mathrm{a}}$ and of $\mathrm{K}^{+}$and $\mathrm{Ca}^{2+}$ in a THF-water mixture, ${ }^{33 \mathrm{~b}}$ was carried out. In these assemblies, however, the main role of the nanocrystal is that of a scaffold for the molecular ligands, not that of signalling unit.

Metal ion sensing based on photoinduced electron transfer was recently realized with CdTe QDs $(d>3 \mathrm{~nm})$ functionalized with ligands consisting of a 1,10-phenanthroline moiety directly linked to azacrown ethers (Fig. 15). ${ }^{34}$ Upon replacement of the native caps with the phenanthroline-azacrown ether ligand, the QD luminescence is quenched because of electron transfer from the phenanthroline HOMO, filling the hole left in the valence band of the photoexcited QD. The addition of $\mathrm{Ba}^{2+}$ ions, which are complexed by the azacrown ether units, makes the oxidation of the phenanthroline moiety more difficult by lowering the HOMO level. As a consequence, the electron-transfer quenching process is prevented and the QD emission strongly increases, thereby providing the basis for luminescent sensing of $\mathrm{Ba}^{2+}$ in organic solution (Fig. 15). ${ }^{34}$

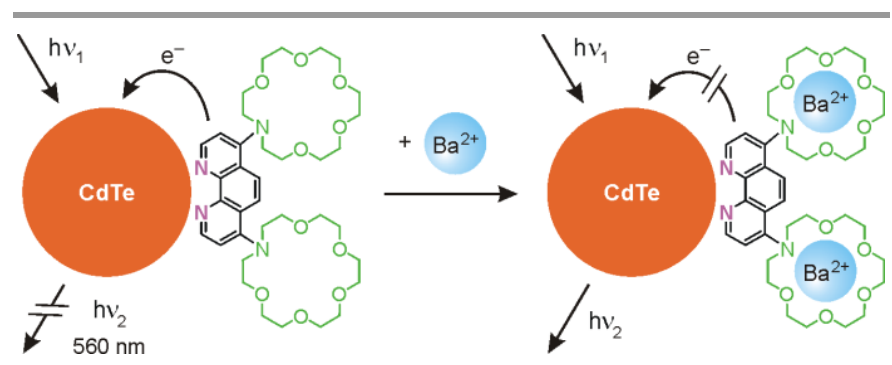

Fig. 15 Luminescent sensing of $\mathrm{Ba}^{2+}$ ions with $\mathrm{CdTe}$ QDs functionalized with phenanthroline-azacrown ether ligands. ${ }^{34}$

The detection of toxic heavy metals with high sensitivity is very important for preventing dangerous effects on health and environment. A ratiometric sensor for $\mathrm{Hg}^{2+}$ based on CdSe-ZnS QDs functionalized with rhodamine $\mathrm{B}$, a dye sensitive to mercury ions, was reported (Fig. 16a). ${ }^{35}$ The hydrophobic nature of the dye causes its non-specific adsorption onto the surface of polymer-encapsulated CdSe-ZnS QDs in water. The pendant thiosemicarbazide group suppresses absorption and emission in the visible region, due to the disruption of the extended aromatic structure of the dye. The desulfurization reaction triggered by $\mathrm{Hg}^{2+}$ ions restores the intense visible absorption of rhodamine $\mathrm{B}$, making it an efficient FRET acceptor for the QD donor. Therefore, the addition of mercury(II) ions causes a decrease in the QD emission $\left(\lambda_{\mathrm{em}}=\right.$ $550 \mathrm{~nm}$ ) and a concomitant increase in the rhodamine fluorescence $\left(\lambda_{\mathrm{em}}=600 \mathrm{~nm}\right)$, forming the basis for the ratiometric response (Fig. 16b). 


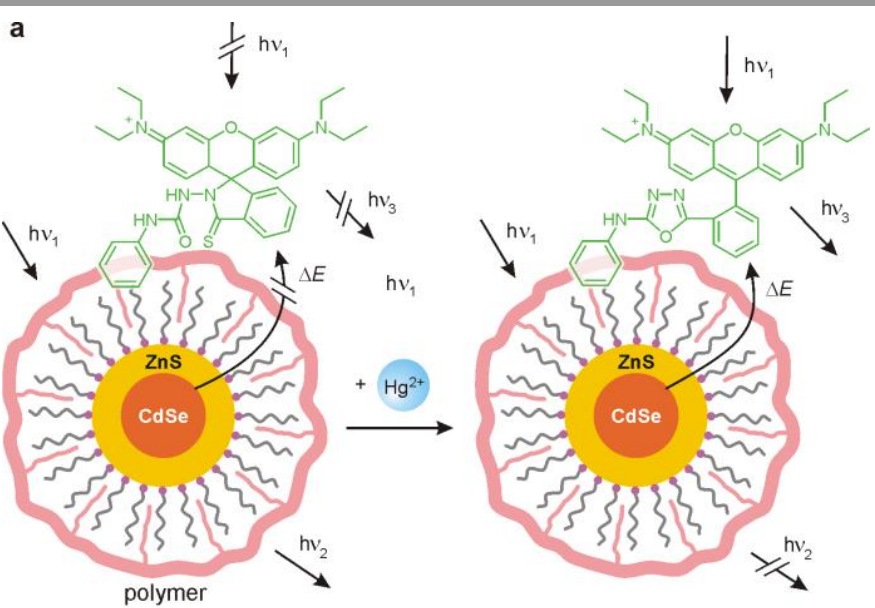

b

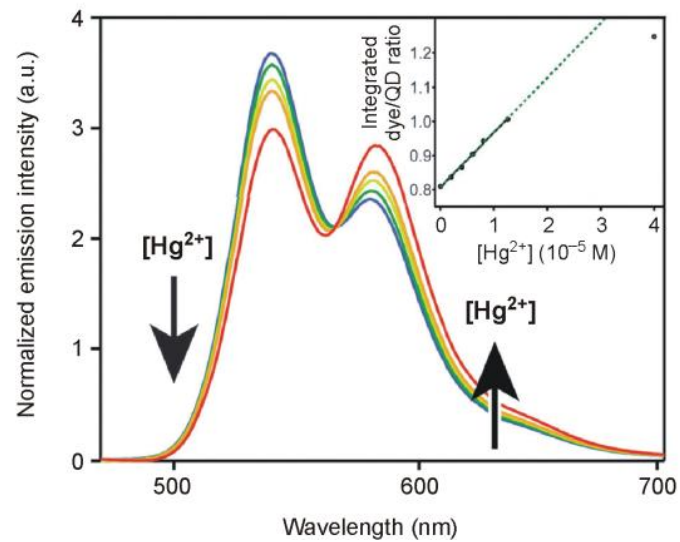

Fig. 16 (a) Schematic mechanism of luminescent sensing of $\mathrm{Hg}^{2+}$ ions with CdSeZnS QDs functionalized with a rhodamine B ligand. ${ }^{35}$ (b) Normalized emission spectra of an aqueous solution of the nanohybrids (dye:QD ratio $=41$ ) at different $\mathrm{Hg}^{2+}$ concentrations (from blue to red, 0 to $4.0 \times 10^{-5} \mathrm{M}$ ). The inset shows the ratiometric response of the sensor. Adapted with permission from ref. 35. Copyright 2011 Royal Society of Chemistry.

FRET between the nanocrystal and a molecular quencher attached by means of a metal-ion specific DNAzyme was exploited to develop high sensitivity, multiplexed detection of $\mathrm{Cu}^{2+}$ and $\mathrm{Pb}^{2+} .{ }^{36}$ The luminescence of carboxyl-silanized CdSe$\mathrm{ZnS}$ QDs is effectively decreased when a FRET quencher is covalently bound to the surface by means of a bridge consisting of a DNA substrate and a DNA enzyme exhibiting high sensitivity and selectivity for $\mathrm{Cu}^{2+}$ or $\mathrm{Pb}^{2+}$ ions (Fig. 17). In the presence of such ions the DNAzymes cleave their substrate, turning on the QD luminescence. Detection limits as low as 0.5 and $0.2 \mathrm{nM}$ for $\mathrm{Cu}^{2+}$ and $\mathrm{Pb}^{2+}$, respectively, are reached nearly 2 orders of magnitude better than the then state-of-theart.

Multiplexed (that is, simultaneous and independent) detection of these two ions was performed using QDs of two different colors, taking advantage of the wide excitation and narrow emission profiles of the nanocrystals (Fig. 17b). Briefly, upon 480-nm excitation of a mixture containing $\mathrm{Pb}^{2+}$-specific $\left(\lambda_{\mathrm{em}}=530 \mathrm{~nm}\right)$ and $\mathrm{Cu}^{2+}$-specific $\left(\lambda_{\mathrm{em}}=625 \mathrm{~nm}\right)$ QDDNAzymes conjugates, $\mathrm{Pb}^{2+}$ and $\mathrm{Cu}^{2+}$ ions could be simultaneously and quantitatively detected with virtually no cross-talk between the $530 \mathrm{~nm}$ and the $625 \mathrm{~nm}$ channels. ${ }^{36}$

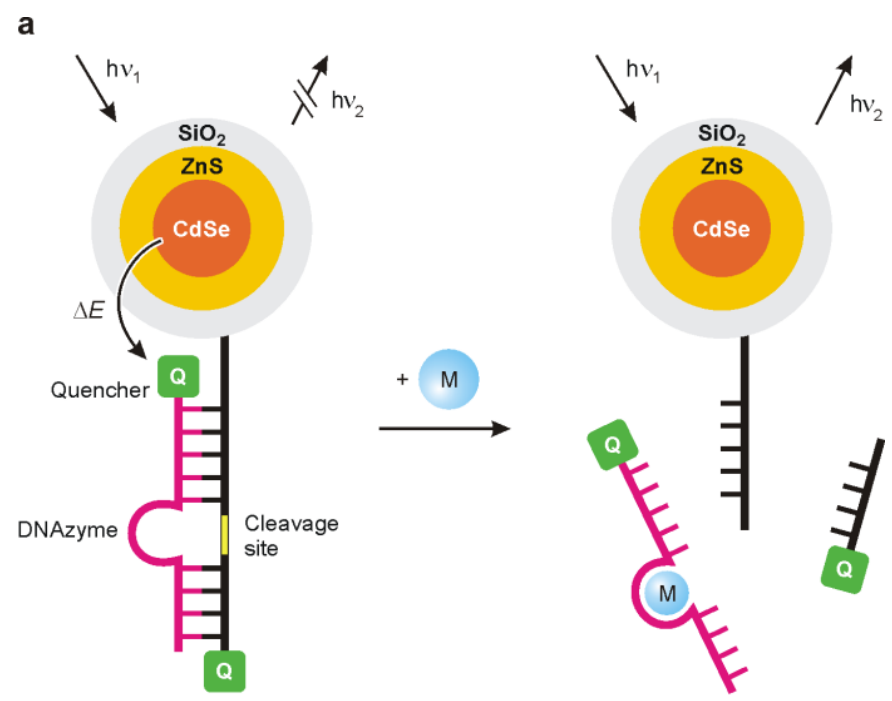

b

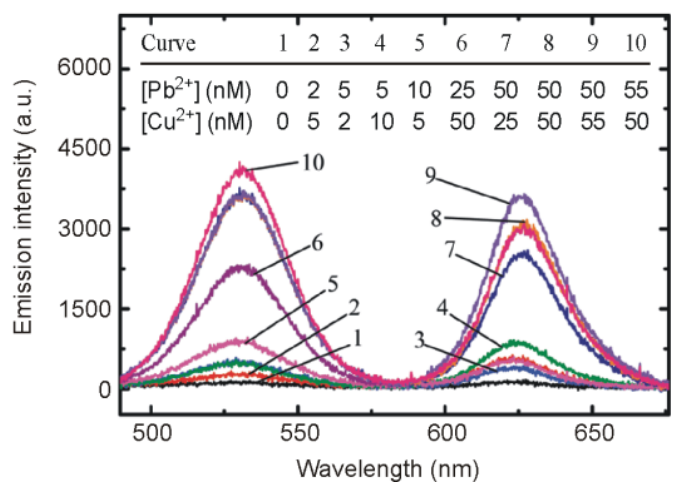

Fig. 17 (a) Schematic representation of luminescent sensing of $\mathrm{Pb}^{2+}$ or $\mathrm{Cu}^{2+}$ with QD-DNAzymes conjugates. ${ }^{36}$ (b) Multiplexed detection of $\mathrm{Pb}^{2+}$ and $\mathrm{Cu}^{2+}$ ions. For more details see the text. Adapted with permission from ref. 36. Copyright 2010 American Chemical Society.

In a related study, the surface of CdSe-ZnS QDs $\left(\lambda_{\mathrm{em}}=560\right.$ $\mathrm{nm}$ ) was modified by covalent attachment of a thymine-rich oligonucleotide (Fig. 18). ${ }^{37}$ In the presence of $\mathrm{Hg}^{2+}$ the oligonucleotide forms a rigid hairpin structure in which the thymine residues are bound to the metal ion. Addition of mercury(II) ions causes a strong quenching of the QD emission, most likely because of an electron transfer from the photoexcited nanocrystal to the bound metal ion. A similar turn-off response is obtained with $\mathrm{CdSe}-\mathrm{ZnS}$ QDs $\left(\lambda_{\mathrm{em}}=620\right.$ $\mathrm{nm}$ ) functionalized with a cytosine-rich oligonucleotide that can selectively bind $\mathrm{Ag}^{+}$ions. Multiplexed luminescent detection of $\mathrm{Hg}^{2+}$ and $\mathrm{Ag}^{+}$was performed, and operation of the system as a logic gate transforming chemical inputs into a luminescent output was demonstrated. 


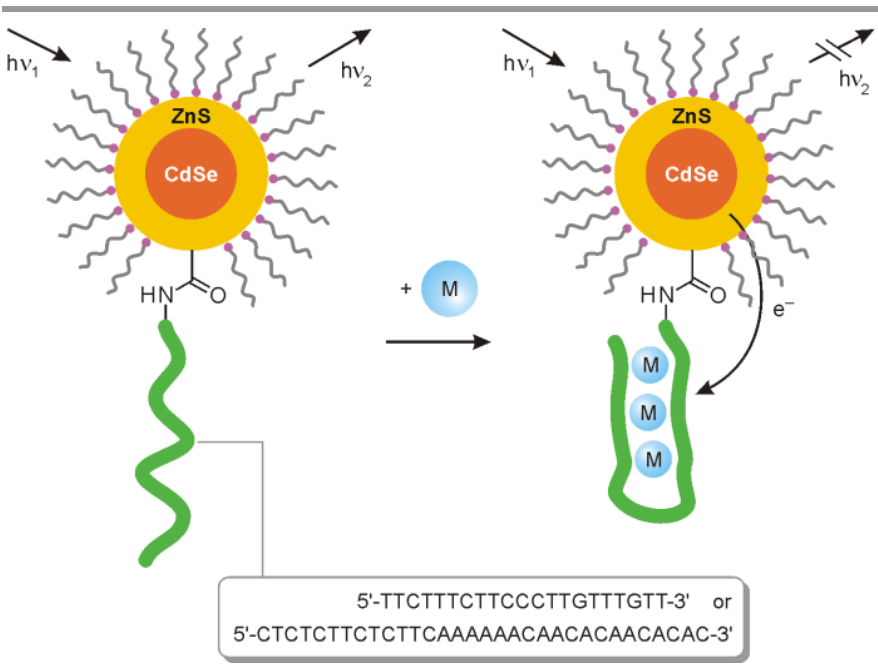

Fig. 18 Schematic representation of luminescent sensing of $\mathrm{Hg}^{2+}$ and $\mathrm{Ag}^{+}$with QD-oligonucleotide conjugates. ${ }^{37}$ For more details see the text.

\section{Anion sensors}

Nanocrystal-based luminescent sensors for anions may be developed by functionalizing the surface of QDs with anion receptors coupled with quenching moieties. A first realization of this strategy is represented by CdSe-ZnS QDs modified with ligands containing a phenyl-thiourea moiety as the anion receptor and a pendant thiol as the surface docking group (Fig. 19). ${ }^{38}$ In chloroform the thiourea-functionalized QDs, obtained by ligand exchange, exhibit the typical nanocrystal luminescence at $\lambda_{\mathrm{em}}=540 \mathrm{~nm}$. Addition of either $\mathrm{Cl}^{-}, \mathrm{F}^{-}$or $\mathrm{CH}_{3} \mathrm{COO}^{-}$ions causes a significant emission quenching. As QDs not bearing the thiourea moiety exhibit no luminescent change in response to the same anions, it can be concluded that the observed effect is due to anion binding to the QD surface mediated by the molecular receptor.

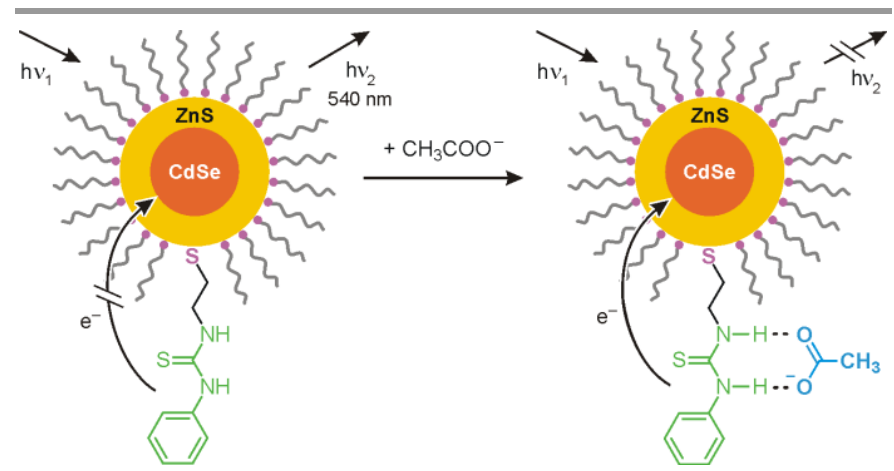

Fig. 19 Schematic mechanism of the turn-off luminescent detection of acetate with a QD-phenyl-thiourea conjugate. ${ }^{38}$

The lack of response for $\mathrm{Br}^{-}$and $\mathrm{HSO}_{4}^{-}$is assigned to inefficient complexation of these anions by the thiourea moiety. Since the receptor, either free or complexed, has no low lying energy levels, a FRET process can be ruled out. Hence, it was hypothesized that quenching arises from electron transfer from the HOMO of the molecule to the QD, activated because anion binding makes the receptor easier to oxidize.
Selective turn-on sensing of carbonate ions was performed with CdSe QDs functionalized with ligands bearing a substituted urea receptor (Fig. 20). ${ }^{39}$ The replacement of the native TOPO capping molecules with the functional ligands causes a strong decrease of the QD luminescence intensity $\left(\lambda_{\mathrm{em}}\right.$ $=583 \mathrm{~nm}$ ), probably because of an electron transfer from the photoexcited QD to the chemisorbed ligand. Complexation of $\mathrm{CO}_{3}{ }^{2-}$ anions by the ureidic moiety of the ligand causes a substantial, albeit incomplete, recovery of the nanocrystal luminescence. It is likely that coordination of the carbonate anions shifts negatively the reduction potential of the ligand and/or increases its rigidity, resulting in the suppression of the electron-transfer quenching. The chemosensor exhibits remarkable selectivity for $\mathrm{CO}_{3}{ }^{2-}$ against several competing anions (halides, nitrate, nitrite, $\mathrm{HPO}_{4}{ }^{2-}, \mathrm{H}_{2} \mathrm{PO}_{4}^{-}, \mathrm{HCO}_{3}^{-}, \mathrm{HSO}_{4}{ }^{-}$ ) but, unfortunately, it operates in organic solution (chloroformethanol 4:1) rather than in water.

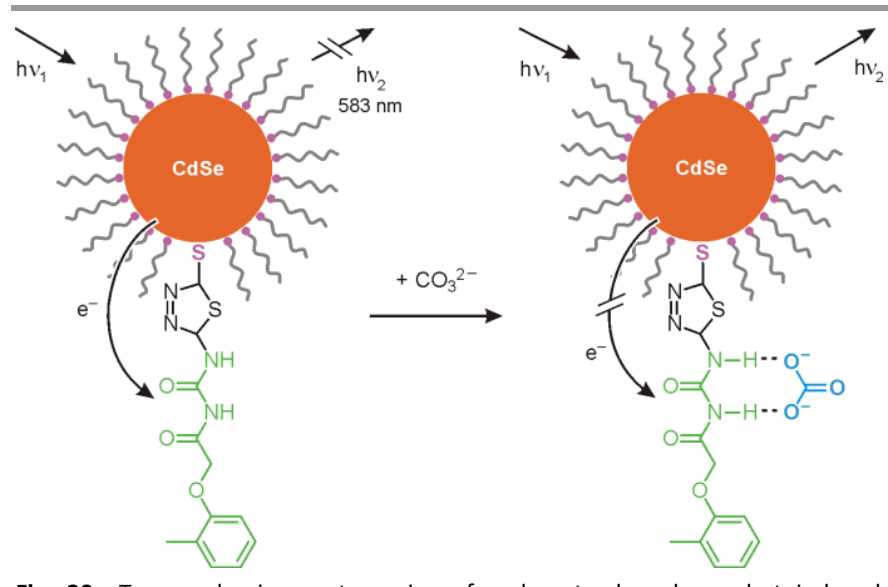

Fig. 20 Turn-on luminescent sensing of carbonate, based on photoinduced electron transfer, with a QD-urea conjugate. ${ }^{39}$

\section{Oxygen sensors}

Oxygen is a most relevant analyte in a variety of applications, ranging from life sciences to environmental and food control. As for $\mathrm{pH}$ sensing, the most common approach for the detection of $\mathrm{O}_{2}$ with semiconductor nanocrystals is based on QDchromophore conjugates. ${ }^{26} \mathrm{CdSe}-\mathrm{ZnS}$ QDs were functionalized with pyrenyl ligands to accomplish ratiometric oxygen sensing (Fig. 21a). ${ }^{40}$ The emission spectrum of the nanohybrid shows both pyrene $\left(\lambda_{\mathrm{em}}=378 \mathrm{~nm}\right)$ and QD spectral features $\left(\lambda_{\mathrm{em}}=\right.$ $632 \mathrm{~nm}$ ), with the two emission signals fully independent from one another. The luminescence spectrum of the nanohybrid is strongly influenced by oxygen concentration; specifically the surface-bound fluorophore is quenched by $\mathrm{O}_{2}$, whereas the QD emission is not (Fig. 21b). Therefore the basis for ratiometric sensing stems from the inherent response of the functional ligand to oxygen and the orthogonal behaviour of the chromophores rather than from a FRET mechanism. 


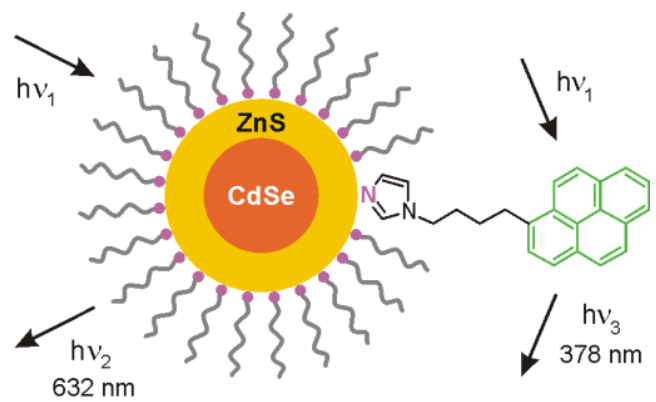

b

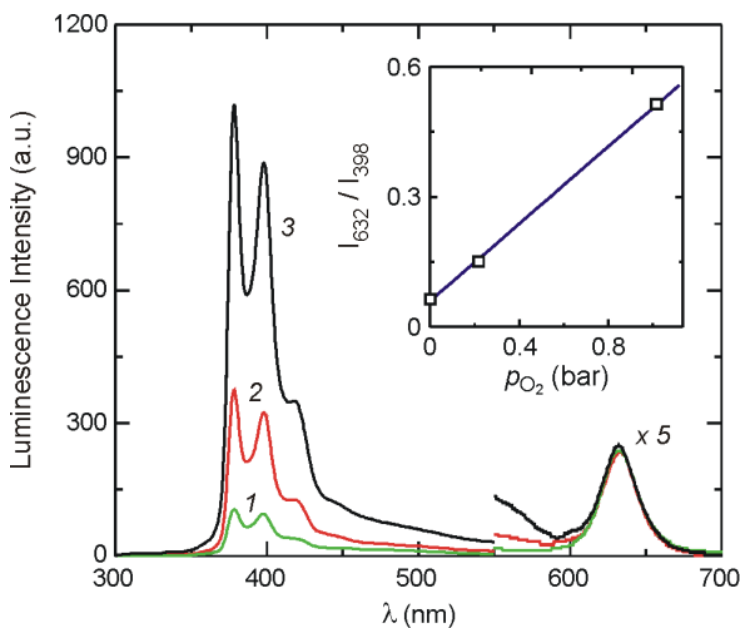

Fig. 21 (a) Schematic representation of CdSe-ZnS QDs (core diameter, $5.8 \mathrm{~nm}$ total diameter, $7.9 \mathrm{~nm}$ ) capped with TOPO and pyrenyl units ${ }^{40}$ (b) Emission spectra $\left(\lambda_{\text {exc }}=275 \mathrm{~nm}\right.$ ) of the nanohybrids in $\mathrm{CHCl}_{3}$ in the presence of: 0 (curve 1), 0.213 (curve 2) and 1.013 (curve 3) bar $\mathrm{O}_{2}$. The inset shows the linear correlation between the ratiometric photoluminescence response (calculated as the QD-to-pyrene emission intensity ratio) and the $\mathrm{O}_{2}$ partial pressure. Reproduced with permission from ref. 40. Copyright 2011 Royal Society of Chemistry.

As oxygen is an analyte of biological interest, near-IR excitation and detection are valuable features of sensors for imaging of live tissues. QDs exhibit large two-photon absorption cross-sections, therefore they are good candidates for multiphoton imaging. Two-photon oxygen sensing with a self-assembled QD-palladium porphyrin conjugate was reported. ${ }^{41}$ Palladium porphyrins are ideal oxygen sensors in the biologically relevant range (0-160 Torr), but they must be combined with an efficient two-photon antenna such as coumarin. The advantage of using QDs instead of an organic dye resides in the large two-photon absorption cross section of QDs (1000-fold greater than common organic dyes), combined with the facile assembly of the nanoconjugate and the tunability of the photophysical properties of the nanocrystals. The emission spectrum of the nanohybrid exhibits features characteristic of both the porphyrin $\left(\lambda_{\mathrm{em}}=690 \mathrm{~nm}\right)$ and the QD $\left(\lambda_{\mathrm{em}}=518 \mathrm{~nm}\right)$. The emission of the porphyrin, however, is more intense when bound to the nanocrystal because it is sensitized by the QD absorption as a result of FRET. Hence, in the assembly the QD serves as an antenna for light absorption: upon selective excitation of the QD, the energy is tranferred to the porphyrin, which then emits (Fig. 22).

The FRET enhancement of the porphyrin emission via the QD energy donor is more pronounced under two-photon excitation, as the two-photon absorption cross-section of the QD is four orders of magnitude greater than that of the porphyrin. As for the previous example, in the conjugate the emission of the chromophore is quenched by oxygen, and the $\mathrm{O}_{2}$-insensitive QD emission serves as an internal standard to afford a ratiometric response.

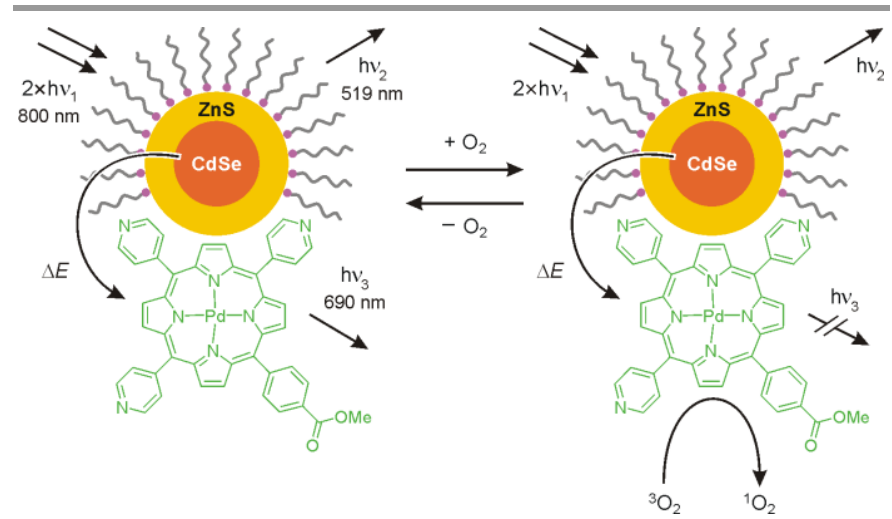

Fig. 22 A QD-Pd porphyrin conjugate in which the QD acts as a two-photon antenna for NIR excitation. The insensitivity of the QD emission to $\mathrm{O}_{2}$ affords an internal reference to establish a ratiometric response to oxygen. ${ }^{41}$

\section{Sensors for other molecular species}

Saccharides are important molecules for which luminescent sensors based on functionalized QDs have been developed. ${ }^{42,43,44,45}$ In one such investigations, a Maltose Binding Protein (MBP) moiety was functionalized with a $\mathrm{Ru}(\mathrm{II})$ (tetraamino)(phenanthroline) complex and attached to thiol hexadecanoate (THDA)-capped CdSe QDs via a metallothionein (T) bridge (Fig. 23). ${ }^{43}$ In the resulting assembly, the ruthenium complex - which is a good electron donor - is sufficiently close to the surface of the nanocrystal to efficiently transfer an electron to the QD upon photoexcitation. This process leads to a substantial quenching of the QD luminescence $\left(\lambda_{\mathrm{em}}=570 \mathrm{~nm}\right)$. Upon maltose binding to $\mathrm{MBP}$, the protein undergoes a significant change in its conformation, moving the ruthenium quencher away from the nanocrystal surface. As a result, the QD emission increases, thus providing an electron-transfer mechanism to transduce the presence of maltose into a luminescence output.

In another study, glutathione-capped CdSe-ZnS QDs were functionalized with methylene blue $\left(\mathrm{MB}^{+}\right)$-modified avidin molecules by covalent coupling of the avidin and glutathione residues (Fig. 24a).45 Methylene blue quenches the QD emission by energy transfer, whereas its reduced form (MBH) does not. Since $\mathrm{MB}^{+}$is reduced by the 1,4-dihydronicotinamide adenide dinucleotide (NADH) cofactor, these QDs can be used as luminescent sensors for NADH (Fig. 24a). The system was then combined with the $\mathrm{NAD}^{+}$-dependent glucose dehydrogenase (GDH) enzyme to implement glucose sensing. 
A biotinylated GDH was linked to the avidin-rich QD surface by exploiting the biotin-avidin interaction. The GDH-catalyzed oxidation of glucose generates NADH, which is subsequently detected by the $\mathrm{MB}^{+}$-functionalized QDs (Fig. 24b).

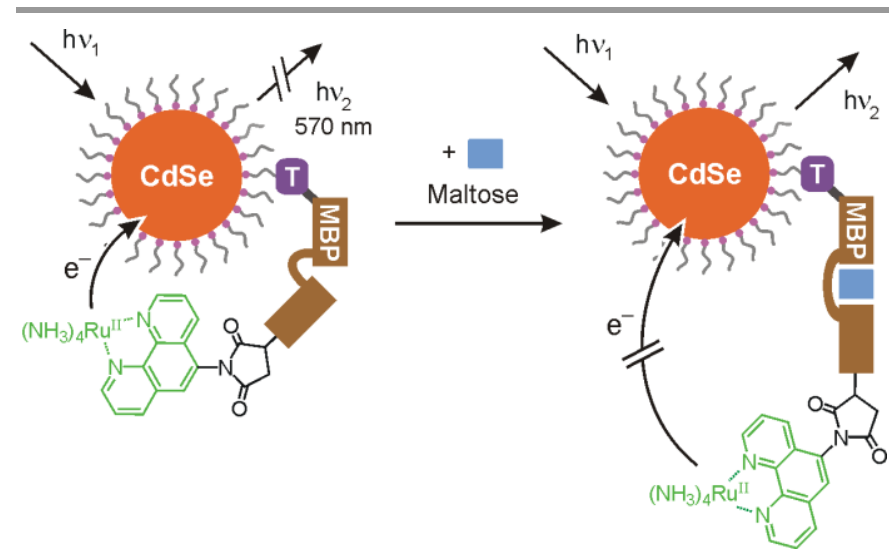

Fig. 23 A CdSe-based sensor for maltose relying on an electron-transfer quenching mechanism. ${ }^{43}$

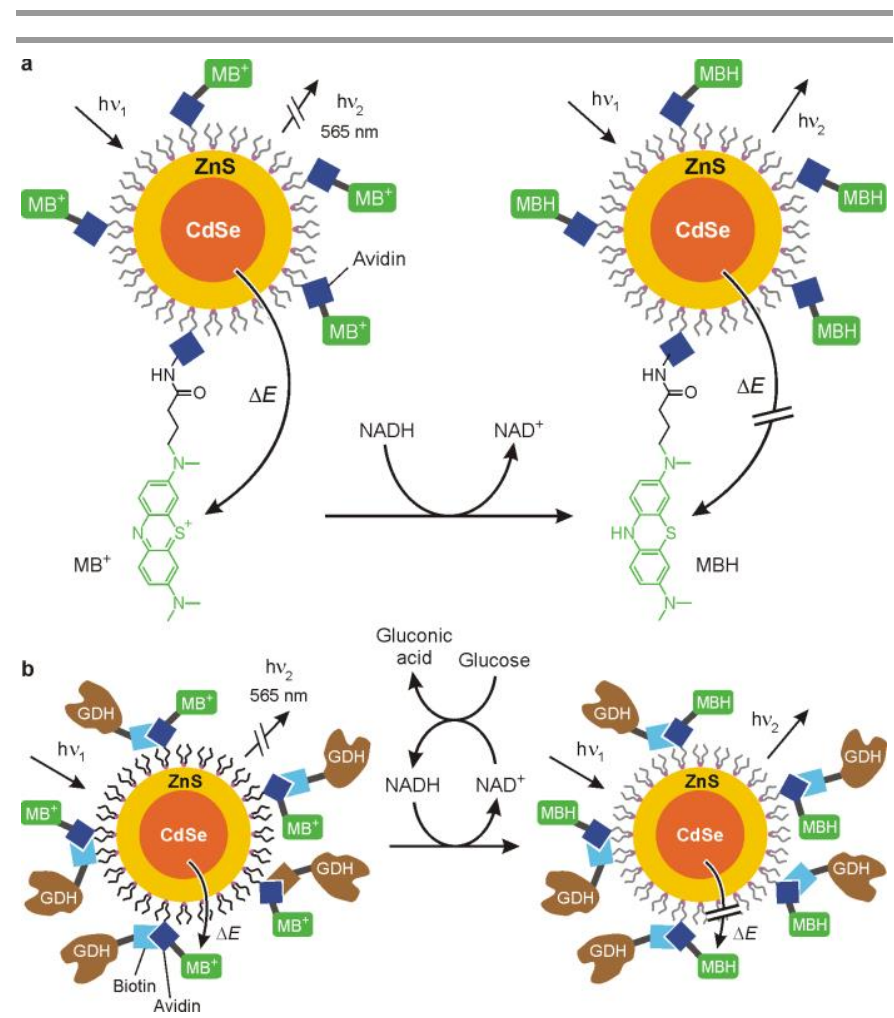

Fig. 24 (a) Sensing of NADH based on energy transfer from CdSe-ZnS QD to methylene blue components in a nanohybrid. (b) Schematic representation of the mechanism for glucose sensing. ${ }^{45}$

CdSe-ZnS core-shell QDs capped with mercaptoacetate anions in solution were exposed to a compound incorporating a 4,4'-bipyridinium electron acceptor and a biotin unit. ${ }^{46}$ The bipyridinium dication adsorbs onto the anionic surface of the QDs and quenches their luminescence by photoinduced electron transfer. If, however, the protein streptavidin is added to the solution, the QD luminescence is increased. This is because streptavidin binds the biotin unit attached to the bipyridinium quencher, which in turn is moved away from the nanocrystal surface. Thus, a protein-ligand complementarity is signalled by a luminescence change.

CdSe-ZnS QDs functionalized with $\beta$-cyclodextrin $(\beta-C D)$ hosts have revealed useful for luminescent sensing of a variety of organic substrates. ${ }^{47}$ For example, the QD emission is quenched by FRET when the $\beta-C D$ units incorporate rhodamine $B$ units (Fig. 25). The intense nanocrystal luminescence is restored in the presence of analytes such as adamantanecarboxylic acid or $p$-hydroxytoluene, because these species displace the FRET quencher from the $\beta-C D$ cavity. As $\beta-C D$ is chiral, discrimination between enantiomeric forms of the same analyte (e.g., L- and D-tyrosine or phenylalanine) can be obtained. ${ }^{47}$
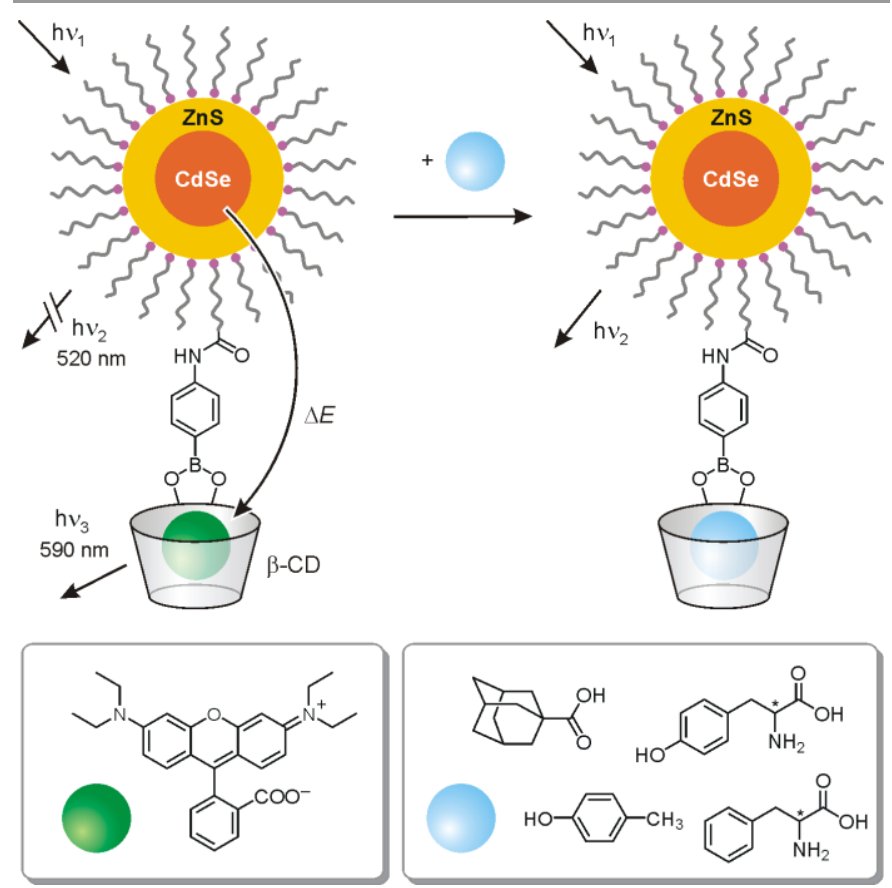

Fig. 25 Luminescent sensing of analytes by a displacement assay using $\beta$ cyclodextrin-modified QDs. ${ }^{47}$

In a related study, a supramolecular assembly based on ketoprofen (KP)-functionalized CdSe-ZnS QDs and pyrenemodified $\beta-C D$ was used for luminescent sensing of analytes such as benzophenone, phenol and indole in acetonitrile/water mixtures (Fig. 26). ${ }^{48}$ The association of the KP-capped CdSe$\mathrm{ZnS}$ QDs with the pyrene-functionalized $\beta$-CD leads to the quenching of the QD emission $\left(\lambda_{\mathrm{em}}=570 \mathrm{~nm}\right)$, presumably because of an electron-transfer process involving the pyrenyl unit. Upon addition of the analyte, which must be a guest for cyclodextrin, the pyrene-decorated $\beta-C D$ is removed from the QD surface and the luminescence of the nanocrystals is enhanced. After the sensing experiment, the QD and $\beta$-CDpyrene components can be recovered upon extraction.

QD-based luminescent sensors for nitroaromatic compounds, including the explosive 2,4,6-trinitrotoluene (TNT), have also been developed. ${ }^{49}$ 
A methodology for multiplexed chemical analysis relying on energy transfer from luminescent terbium complexes to different QDs, established by molecular recognition between streptavidin and biotin, was proposed. ${ }^{50}$ This strategy is transferable to real-world diagnostic assays that use RNA-, DNA-, aptamer-, peptide- or protein-based recognition, and was recently utilized for the determination of prostate specific antigen (PSA) in serum with sub-nM detection limit. ${ }^{51}$

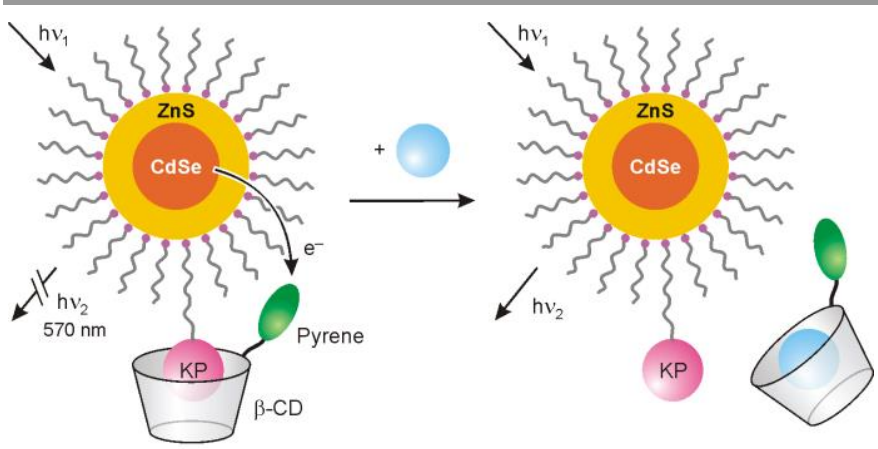

Fig. 26 Luminescent sensing of molecular species by a displacement assay using ketoprofen-modified QDs and pyrene-decorated $\beta$-cyclodextrin. ${ }^{48}$

\section{Summary and outlook}

Progress in the preparation and surface modification of monodisperse semiconductor nanocrystals with predetermined optical properties has recently enabled the use of quantum dots as the luminescent components for the modular construction of sensing nanoassemblies. These systems exhibit an emission signal, coming from the inorganic nanocrystal, that can be modulated by surface-attached chemo-sensitive molecular species by relying on energy- or electron-transfer mechanisms.

Semiconductor nanocrystals are superior to organic dyes in this regard not only because of their peculiar size-dependent optical properties but also because of their excellent photostability and the possibility to act as scaffolds for arranging functional molecular units on their surface. A number of strategies to control and modulate the properties of QDs including long-term stability, solubility and biocompatibility while maintaining a size in the order of a few nanometers and preserving their outstanding optical and electronic properties have become available in the past few years.

However, despite much progress in the area of QDs, particularly for CdSe-ZnS particles, several aspects of the physical and chemical behavior of these nanomaterials are not yet satisfactorily understood and therefore further theoretical and experimental advancements are needed. The irreproducibility issues inherent to the preparation of QDs, the erratic behaviour of their surface, and the artifacts associated with their characterization are significant obstacles for the rational design and construction of QD-based systems. In this context, basic investigations on the physico-chemical behavior of semiconductor nanocrystals in relation to their structure and preparation method are desirable. Finally, nanocrystal toxicity and environmental problems arising from the use of cadmium- based semiconducting materials are still elements of concern in the utilization of QD sensors in the real world. All these stimulating challenges can be addressed with a multidisciplinary research effort, ultimately enabling a full technological exploitation of such fascinating nanodevices.

\section{Acknowledgments}

Financial support from the Italian Ministery of Education, University and Research (PRIN 2010CX2TLM “InfoChem"), the Université Franco-Italienne (Vinci programme) and the University of Bologna is gratefully acknowledged.

\section{Notes and references}

${ }^{a}$ Photochemical Nanosciences Laboratory and Center for the Chemical Conversion of Solar Energy (SolarChem), Dipartimento di Chimica "G. Ciamician", via Selmi 2, 40126 Bologna, Italy. Fax: +39 051 2099456; Tel: +39 051 2099540; E-mail: alberto.credi@unibo.it; Web: www.credigroup.it

1 (a) Al. L. Efros, A. L. Efros, Sov. Phys. Semicond., 1982, 16, 772775; (b) L. E. Brus, J. Chem. Phys., 1983, 79, 5566-5571.

2 C. B. Murray, D. J. Norris, M. G. Bawendi, J. Am. Chem. Soc., 1993, 115, 8706-8715.

3 (a) U. Resch-Genger, M. Grabolle, S. Cavaliere-Jaricot, R. Nitschke and T. Nann, Nat. Methods, 2008, 5, 763-775; (b) B. Hötzer, I. L. Medintz and N. Hildebrandt, Small, 2012, 8, 2297-2326.

4 (a) T. Pellegrino, S. Kudera, T. Liedl, A. Muñoz-Javier, L. Manna and W. J. Parak, Small, 2005, 1, 48-63; (b) X. Michalet, F. F. Pinaud, L. A. Bentolila, J. M. Tsay, S. Doose, J. J. Li, G. Sundaresan, A. M. Wu, S. S. Gambhir and S. Weiss, Science, 2005, 307, 538-544.

5 See, e.g.: (a) D. V. Talapin, J. S. Lee, M. V. Kovalenko and E. V. Shevchenko, Chem. Rev., 2010, 110, 389-458; (b) P. V. Kamat, K. Tvrdy, D. R. Baker and J. G. Radich, Chem. Rev., 2010, 110, 66646688; (c) C. de Mello Donegá, Chem. Soc. Rev., 2011, 40, 15121546; (d) T. L. Doane and C. Burda, Chem. Soc. Rev., 2012, 41, 2885-2911; (e) M. Amelia, C. Lincheneau, S. Silvi and A. Credi, Chem. Soc. Rev., 2012, 41, 5728-5743; (f) Y. Shirasaki, G. J. Supran, M. G. Bawendi and V. Bulović, Nat. Photon., 2013, 7, 13-23; (g) M. J. Fernée, P. Tamarat and B. Lounis, Chem. Soc. Rev., 2014, 43, 1311-1337; (h) T. Avellini, C. Lincheneau, F. Vera, S. Silvi and A. Credi, Coord. Chem. Rev., 2014, 263-264, 151-160.

6 (a) Nanoparticles: from Theory to Applications, G. Schmid, ed., Wiley-VCH, Weinheim, 2004; (b) Semiconductor and Metal Nanocrystals, V. I. Klimov, ed., Dekker, New York, 2005; (c) Semiconductor Nanocrystal Quantum Dots, A. L. Rogach, ed., Springer-Verlag, Wien, 2008; (d) Quantum Dot Sensors: Technology and Commercial Applications, J. F. Callan and F. M. Raymo, eds., Pan Stanford Publishing, Singapore, 2013.

7 A. P. de Silva, H. Q. N. Gunaratne, T. Gunnlaugsson, N. A. J. M. Huxley, C. P. McCoy, J. T. Rademacher and T. E. Rice, Chem. Rev., 1997, 97, 1515-1566.

8 (a) R. C. Somers, M. G. Bawendi and D. G. Nocera, Chem. Soc. Rev., 2007, 36, 579-591; (b) F. M. Raymo and I. Yildiz, Phys. Chem. Chem. Phys., 2007, 9, 2036-2042; (c) J. Callan, A. P. De Silva, R. C. 
Mulrooney and B. McCaughan, J. Incl. Phenom. Macroc. Chem., 2007, 58, 257-262; (d) R. Freeman and I. Willner, Chem. Soc. Rev., 2012, 41, 4067-4085; (e) E. Petryayeva, W. R. Algar and I. L. Medintz, Appl. Spectr., 2013, 67, 215-252; (f) P. Wu, T. Zhao. S. Wang and X. Hou, Nanoscale, 2014, 6, 43-64; (g) C. M. Tyrakowski and P. T. Snee, Phys. Chem. Chem. Phys., 2014, 16, 837-855.

9 Y. Chen and Z. Rosenzweig, Anal. Chem., 2002, 74, 5132-5138.

10 M. Green, J. Mater. Chem., 2010, 20, 5797-5809.

11 P. Reiss, M. Protière and L. Li, Small, 2009, 5, 154-168.

12 M. Amelia, S. Impellizzeri, S. Monaco, I. Yildiz, S. Silvi, F. M. Raymo and A. Credi, ChemPhysChem, 2011, 12, 2280-2288.

13 V. Lesnyak, N. Gaponik and A. Eychmüller, Chem. Soc. Rev., 2013, 42, 2905-2929.

14 S. J. Byrne, S. A. Corr, T. Y. Rakovich, Y. K. Gun'ko, Y. P. Rakovich, J. F. Donegan, S. Mitchell and Y. Volkov, J. Mater. Chem., 2006, 16, 2896-2902.

15 G. Palui, F. Aldeek, W. Wang and H. Mattoussi, Chem. Soc. Rev., 2014, DOI: 10.1039/c4cs00124a.

16 See, e.g.: F. Wang, R. Tang, J. L.-F. Kao, S. D. Dingman and W. E. Buhro, J. Am. Chem. Soc., 2009, 131, 4983-4994.

17 (a) I. L. Medintz, H. T. Uyeda, E. R. Goldman and H. Mattoussi, Nat. Mater., 2005, 4, 435-446; (b) N. Erathodiyil and J. Y. Ying, Acc. Chem. Res., 2011, 44, 925-935; (c) T. Avellini, C. Lincheneau, M. La Rosa, A. Pertegás, H. J. Bolink, I. A. Wright, E. C. Constable, S. Silvi and A. Credi, Chem. Commun., 2014, 50, 11020-11022.

18 W. Liu, M. Howarth, A. B. Greytak, Y. Zheng, D. G. Nocera, A. Y. Ting and M. G. Bawendi, J. Am. Chem. Soc., 2008, 130, 1274-1284.

19 I. Yildiz, E. Deniz, B. McCaughan, S. F. Cruickshank, J. F. Callan and F. M. Raymo, Langmuir, 2010, 26, 11503-11511.

20 See e.g. Chem. Soc. Rev., 2014, 43, 3995-4270 (themed issue on photochemistry of supramolecular systems and nanostructured assemblies).

21 V. Balzani, A. Credi and M. Venturi, Molecular Devices and Machines - Concepts and Perspectives for the Nano World, WileyVCH, Weinheim, 2008.

22 D. Rehm and A. Weller, Isr. J. Chem., 1970, 8, 259-263.

23 J. R. Lakowicz, Principles of Fluorescence Spectroscopy, 3rd Edition, Springer, New York, 2006.

24 (a) I. Yildiz, M. Tomasulo and F. M. Raymo, J. Mater. Chem., 2008, 18, 5577-5584; (b) I. L. Medintz and H. Mattoussi, Phys. Chem. Chem. Phys., 2009, 11, 17-45.

25 T. Avellini, M. Amelia, A. Credi and S. Silvi, Langmuir, 2013, 29, 13352-13358.

26 C. M. Lemon, P. N. Curtin, R. C. Somers, A. B. Greytak, R. M. Lanning, R. K. Jain, M. G. Bawendi and D. G. Nocera, Inorg. Chem., 2014, 53, 1900-1915 and references therein.

27 M. Tomasulo, I. Yildiz, S. L. Kaanumalle and F. M. Raymo, Langmuir, 2006, 22, 10284-10290.

28 P. T. Snee, R. C. Somers, G. Nair, J. P. Zimmer, M. G. Bawendi and D. G. Nocera, J. Am. Chem. Soc., 2006, 128, 13320-13321.

29 R. Tang, H. Lee and S. Achilefu, J. Am. Chem. Soc., 2012, 134, 45454548.
30 I. L. Medintz, M. H. Stewart, S. A. Trammell, K. Susumu, J. B. Delehanty, B. C. Mei, J. S. Melinger, J. B. Blanco-Canosa, P. E. Dawson and H. Mattoussi, Nat. Mater., 2010, 9, 676-684.

31 A. M. Dennis, W. J. Rhee, D. Sotto, S. N. Dublin and G. Bao, ACS Nano, 2012, 6, 2917-2924.

32 C.-Y. Chen, C.-T. Cheng, C.-W. Lai, P.-W. Wu, K.-C. Wu, P.-T. Chou, Y.-H. Chou and H.-T. Chiu, Chem. Commun., 2006, 263-265.

33 (a) N. Singh, R. C. Mulrooney, N. Kaur and J. F. Callan, Chem. Commun., 2008, 4900-4902; (b) N. Singh, R. C. Mulrooney, N. Kaur and J. F. Callan, J. Fluoresc., 2009, 19, 777-782.

34 J. Völker, X. Zhou, X. Ma, S. Flessau, H. Lin, M. Schmittel and A. Mews, Angew. Chem. Int. Ed., 2010, 49, 6865-6868.

35 L. E. Page, X. Zhang, A. M. Jawaid and P. T. Snee, Chem. Commun., 2011, 47, 7773-7775.

36 C.-S. Wu, M. K. K. Oo and X. Fan, ACS Nano, 2010, 4, 5897-5904.

37 R. Freeman, T. Finder and I. Willner, Angew. Chem. Int. Ed., 2009, 48, 7818-7821.

38 J. F. Callan, R. C. Mulrooney, S. Kamila and B. McCaughan, J. Fluoresc., 2008, 18, 527-532.

39 C. Han, Z. Cui, Z. Zou, Sabahaiti, D. Tian and H. Li, Photochem. Photobiol. Sci., 2010, 9, 1269-1273.

40 M. Amelia, A. Lavie-Cambot, N. D. McClenaghan and A. Credi, Chem. Commun., 2011, 47, 325-327.

41 C. M. Lemon, E. Karnas, M. G. Bawendi and D. G. Nocera, Inorg. Chem., 2013, 52, 10394-10406.

42 I. L. Medintz, A. R. Clapp, H. Mattoussi, E. R. Goldman, B. Fisher and J. M. Mauro, Nat. Mater., 2003, 2, 630-638.

43 M. G. Sandros, D. Gao and D. E. Benson, J. Am. Chem. Soc., 2005, 127, 12198-12199

44 R. Freeman, L. Bahshi, T. Finder, R. Gill and I. Willner, Chem. Commun., 2009, 764-766

45 L. Bashi, R. Freeman, R. Gill and I. Willner, Small, 2009, 5, 676-680.

46 I. Yildiz, M. Tomasulo and F. M. Raymo, Proc. Natl. Acad. Sci. U.S.A., 2006, 103, 11457-11460.

47 R. Freeman, T. Finder, L. Bahshi and I. Willner, Nano Lett., 2009, 9 , 2073-2076.

48 J. Aguilera-Sigalat, J. M. Casas-Solvas, M. C. Morant-Miñana, A. Vargas-Berenguel, R. E. Galian and J. Pérez-Prieto, Chem. Commun., 2012, 48, 2573-2575.

49 E. R. Goldman, I. L. Medintz, J. L. Whitley, A. Hayhurst, A. R. Clapp, H. T. Uyeda, K. R. Deschamps, M. E. Lassman and H. Mattoussi, J. Am. Chem. Soc., 2005, 127, 6744-6751.

50 D. Geissler, L. J. Charbonnière, R. F. Ziessel, N. G. Butlin, H.-G. Löhmannsröben and N. Hildebrandt, Angew. Chem. Int. Ed., 2010, 49, 1396-1401.

51 K. D. Wegner, Z. Jin, S. Lindén, T. L. Jennings and N. Hildebrandt, ACS Nano, 2013, 7, 7411-7419. 